\title{
Non-autonomous nonlocal partial differential equations with delay and memory
}

\author{
Jiaohui Xu, Zhengce Zhang \\ School of Mathematics and Statistics, Xi'an Jiaotong University, \\ Xi'an, 710049, PR China \\ Tomás Caraballo* \\ Dpto. Ecuaciones Diferenciales y Análisis Numérico, Facultad de Matemáticas, \\ Universidad de Sevilla, c/ Tarfia s/n, 41012-Sevilla, Spain
}

\begin{abstract}
The paper addresses a kind of non-autonomous nonlocal parabolic equations when the external force contains hereditary characteristics involving bounded and unbounded delays. First, well-posedness of the problem is analyzed by the Galerkin method and energy estimations in the phase space $\mathbf{C}_{\rho}(X)$. Moreover, some results related to strong solutions are proved under suitable assumptions. The existence of stationary solutions is then established by a corollary of the Brower fixed point theorem. By constructing appropriate Lyapunov functionals in terms of the characteristic delay terms, a deep analysis on stability and attractiveness of the stationary solutions is established. Furthermore, the existence of pullback attractors in $L^{2}(\Omega)$, with bounded and unbounded delays, is shown. We emphasize that, to prove the existence of pullback attractors in the unbounded delay case, a new phase space, $E_{\gamma}$, has to be constructed.

Keywords: Non-autonomous nonlocal parabolic equations; Bounded and unbounded delays; Stationary solutions; Pullback attractors.
\end{abstract}

\section{Introduction}

It is well-known reaction-diffusion equations have been frequently used to model a great amount of phenomena in the real world. However, in recent decades, nonlocal (reaction-diffusion) problems have been investigated with great interest due to its usefulness in real applications (e.g. $[1,3,16,20,26])$.

The nonlocal character in the equations has different sources. For example, the model in [26] focused on the role of nonlocal (integral) terms describing the interactions between cancer cells and the host tissue. The authors in [4] studied nonlocal problems that are analogous to the local ones given by the Laplacian or the $p$-Laplacian with dynamical boundary conditions.

\footnotetext{
${ }^{*}$ Corresponding author.

E-mail addresses: xujh2009@stu.xjtu.edu.cn (J. Xu), zhangzc@mail.xjtu.edu.cn (Z. Zhang),

caraball@us.es (T. Caraballo.)
} 
One typical nonlocal problem, motivated by the mathematical modeling of a variety of phenomena coming from industrial applications ([27]), and shear banding formation in high strain metals ([3]), etc., has been studied by N. I. Kavallaris et al. [20]. The authors considered the following nonlocal stochastic parabolic problem

$$
\begin{cases}\frac{\partial u}{\partial t}=\Delta u+F(u)+\sigma(u) \partial_{t} W(x, t), & (x, t) \in D_{T}:=D \times(0, T), \\ u(x, t)=0, & (x, t) \in \partial D \times(0, T), \\ u(x, t)=\xi(x), & x \in D,\end{cases}
$$

where $T>0$ and $D$ is a bounded subset of $\mathbb{R}^{d}, d \geq 1$, with smooth boundary. Here the nonlocal term $F(u)$ is defined by

$$
F(u):=\frac{\lambda e^{u}}{\left(\int_{D} f(u) d x\right)^{q}}, \quad q>0,
$$

for some positive constant $\lambda$.

The authors examined in [17] one model of single-species dynamics which incorporates nonlocal effects, comparing with the standard approach to model a single species domain $\Omega$ of "Kolmogorov" type,

$$
u_{t}=\Delta u+\lambda u g(u), \quad \text { in } \Omega, t>0 .
$$

If we take into account the following backgrounds: (i) a population in which individuals compete for a shared rapidly equilibrate resource; (ii) a population in which individuals communicate either visually or by chemical means, then the most straightforward way of introducing nonlocal effects is to consider, instead of $g(u)$, a "crowding" effect of the form $g(u, \bar{u})$, where

$$
\bar{u}(x, t)=\int_{\Omega} G(x, y) u(y, t) d y,
$$

and $G(x, y)$ is some reasonable kernel.

Heuristically, Chipot et al. studied in [13] the behavior of a population of bacteria with nonlocal term $a\left(\int_{\Omega} u\right)$ in a container. Later, Chipot et al. extended this term to a general nonlocal operator $a(l(u))$ (cf. $[11,12,13,14])$, where $l \in \mathcal{L}\left(L^{2}(\Omega) ; \mathbb{R}\right)$, for instance, if $g \in L^{2}(\Omega)$,

$$
l(u)=l_{g}(u)=\int_{\Omega} g(x) u(x) d x .
$$

Then, the following nonlocal reaction-diffusion equation has received much attention due to its wide application (such as, in the ecological context, the interactions in single-species population dynamics cannot be local),

$$
\frac{\partial u}{\partial t}-a(l(u)) \Delta u=f(u)
$$

where the function $a \in C\left(\mathbb{R} ; \mathbb{R}^{+}\right)$, and it should fulfil natural conditions of non-degeneracy to avoid the extinction and the existence of solutions only in finite time intervals. Therefore, assume there exist positive constants $m, M>0$ such that

$$
0<m \leq a(s) \leq M, \quad \forall s \in \mathbb{R}
$$


Amongst the many notable results regarding such kind of nonlocal equations, it is worth stressing the importance of the work in [10]. In this paper, the authors studied a non-autonomous nonlocal parabolic equation similar to (1.1) but with an extra time-dependent term $h(t)$, which allows to model more complex situations.

On the other hand, in real life, time delays can arise everywhere, since every process, whether it is long or short, would consume time. As a motivation, we can mention a simple situation concerning the hemodynamical behavior of a person who has a decompensation of the glucose level (either high or low). Then, this person can be prescribed some intravenous insulin to compensate the level. Since the introduction of the drug in the bloodstream needs time to take effect, the above situation can be interpreted as a delay problem. Therefore, there is an increasing interest in studying biological, physical models with delay, for example, see $[2,6,7,8,9,21,22,25,28]$ and the references therein.

Consequently, in this context it is natural to take into account the effect of delay in the investigation of non-autonomous nonlocal partial differential equations, which leads us to study the following problem:

$$
\begin{cases}\frac{\partial u}{\partial t}-a(l(u)) \Delta u=f(u)+h\left(t, u_{t}\right) & \text { in } \Omega \times[\tau, \infty), \\ u=0 & \text { on } \partial \Omega \times[\tau, \infty), \\ u_{\tau}(x, t)=\varphi(x, t) & \text { in } \Omega \times(-\rho, 0],\end{cases}
$$

where $\Omega \subset \mathbb{R}^{N}$ is a bounded open set, $\tau \in \mathbb{R}$, function $a \in C\left(\mathbb{R} ; \mathbb{R}^{+}\right)$is locally Lipschitz fulfilling (1.2), $f \in C(\mathbb{R})$ and $h$ contains hereditary characteristics involving delays. Moreover, $0<\rho \leq \infty$, which implies, we consider both cases, bounded and unbounded delays in this manuscript.

From the technical point of view, some new difficulties appear with respect to all these works. Due to the peculiarities of delay problems, we have to work in a phase space $\mathbf{C}_{\rho}(X)$ or $E_{\gamma}$ which require a much more involved analysis to prove asymptotic compactness. Thanks to the Ascoli-Arzelà theorem and energy estimations, we could prove long-time behavior successfully.

It is also worth observing that variable delay, distributed delay and long term-memory cases can be contained in our functional delay term $h\left(t, u_{t}\right)$. In fact, the authors in [9] worked on the following reaction-diffusion equation with memory:

$$
\frac{\partial u}{\partial t}-\Delta u+\int_{-\infty}^{t} \gamma(t-s) \Delta u(x, s) d s+g(x, t, u(x, t))=f_{1}(x, t, u(x, t-h))
$$

with Dirichlet boundary condition, and $\gamma$ possesses the special form $\gamma(t)=-\gamma_{0} e^{-d_{0} t}$. In this way the long-time behavior of solutions can be analyzed. However, if we consider a generalized kernel with singularity, $\gamma(t)=\frac{e^{-d_{0} t}}{t^{\alpha}}$, where $\alpha \in(0,1)$, the long-time behavior of this problem cannot be handled with our analysis.

One of our main purposes is to prove existence, uniqueness and regularity properties of weak solutions to problem (1.3). To this end, the well-known Galerkin method is adapted to our 
problem properly. Moreover, we are able to analyze some stability properties (cf. Definition 3.3) of stationary solutions to problem (1.3), when the delay term has special forms (variable delay/ distributed delay). By a direct approach or constructing Lyapunov functionals, our goals are achieved successfully. At last, to make our analysis more complete, we establish the existence of pullback attractors in $L^{2}(\Omega)$ in the bounded and unbounded delay cases. We want to emphasize that, due to the lack of asymptotic compactness for the unbounded delay case, it is impossible to study the existence of pullback attractors in $L^{2}(\Omega)$ if the phase space is defined by $\mathbf{C}_{\infty}\left(L^{2}(\Omega)\right)$. Instead, the existence of pullback attractor is proved considering the phase space $E_{\gamma}$.

This paper is structured as follows. Section 2 is devoted to the existence, uniqueness and continuous dependence on initial values of weak solutions to (1.3) by the Galerkin method. The existence of strong solutions is also shown by energy estimations. In Section 3, existence and uniqueness of stationary solutions are established and several methods are used to analyze the stability of the stationary solutions. We consider the existence of pullback attractors in the last section, providing more details about their structure in the bounded delay case.

\section{Well-posedness to nonlocal partial differential equations with delay}

Here we consider the following nonlocal parabolic equation with bounded or unbounded delay,

$$
\begin{cases}\frac{\partial u}{\partial t}-a(l(u)) \Delta u=f(u)+h\left(t, u_{t}\right) & \text { in } \quad \Omega \times[\tau, \infty), \\ u=0 & \text { on } \partial \Omega \times[\tau, \infty),\end{cases}
$$

with initial value

$$
u(x, \tau+\theta)=\varphi(x, \theta), \quad \theta \in(-\rho, 0], \quad x \in \Omega,
$$

where $\Omega \subset \mathbb{R}^{N}$ is a bounded open set and the initial time $\tau \in \mathbb{R}$. The function $a \in C\left(\mathbb{R} ; \mathbb{R}_{+}\right)$and there exists a constant $m$ such that

$$
0<m \leq a(s), \quad \forall s \in \mathbb{R} .
$$

In addition, we assume that $l \in \mathcal{L}\left(L^{2}(\Omega), \mathbb{R}\right), f \in C(\mathbb{R})$ and there exist constants $\eta>0, C_{f}>0$ such that

$$
\begin{gathered}
|f(s)| \leq C_{f}(1+|s|), \quad \forall s \in \mathbb{R}, \\
(f(s)-f(r))(s-r) \leq \eta(s-r)^{2}, \quad \forall s, r \in \mathbb{R} .
\end{gathered}
$$

Consider a fixed $T>0$, given a function $u:(\tau-\rho, T] \rightarrow L^{2}(\Omega)$, for each $t \in(\tau, T]$, we denote by $u_{t}$ the function defined on $(-\rho, 0]$ via the relation

$$
u_{t}(s)=u(t+s), \quad s \in(-\rho, 0],
$$

where $0<\rho \leq \infty$. 
In order to state the problem in the correct framework, let us first establish some suitable assumptions on the term where the delay is present. In a general way, let $X$ and $Y$ be two separable Banach spaces, and

$$
\mathbf{C}_{\rho}(X)=\left\{\phi \in C((-\rho, 0] ; X) ; \lim _{s \rightarrow-\rho} \phi(s) \text { exists in } X\right\}
$$

which is a Banach space with the standard sup norm $\|\alpha\|_{\mathbf{C}_{\rho}(X)}=\sup _{-\rho \leq \theta \leq 0}\|\alpha(\theta)\|_{X}$, and let $h: \mathbb{R} \times \mathbf{C}_{\rho}(X) \rightarrow Y$, satisfy

$\left(H_{1}\right)$ for all $\xi \in \mathbf{C}_{\rho}(X)$, the mapping $t \in(\tau, T] \rightarrow h(t, \xi) \in X$ is measurable;

$\left(H_{2}\right)$ For each $t \in(\tau, T], h(t, 0)=0$;

$\left(H_{3}\right)$ There exists $L_{h}>0$ such that for all $t \in(\tau, T], \alpha, \beta \in \mathbf{C}_{\rho}(X)$,

$$
\|h(t, \alpha)-h(t, \beta)\|_{Y} \leq L_{h}\|\alpha-\beta\|_{\mathbf{C}_{\rho}(X)}
$$

$\left(H_{4}\right)$ There exists $C_{h}>0$ such that for all $t \in(\tau, T], u, v \in C((\tau-\rho, T] ; X)$,

$$
\int_{\tau}^{t}\left\|h\left(s, u_{s}\right)-h\left(s, v_{s}\right)\right\|_{Y}^{2} d s \leq C_{h} \int_{\tau-\rho}^{t}\|u(s)-v(s)\|_{X}^{2} d s .
$$

Remark 2.1 (i) In order to simplify notation, we denote $\mathbf{C}_{\rho}(X):=C((-\rho, 0] ; X)$, which represents: when it is bounded delay, $\mathbf{C}_{\rho}(X):=C([-\rho, 0] ; X)$; when it is unbounded delay, $\mathbf{C}_{\rho}(X):=$ $C((-\infty, 0] ; X)$.

(ii) Notice that when we consider bounded delay, namely $\rho>0$ is a constant, then the space $\mathbf{C}_{\rho}(X):=\{\phi \in C([-\rho, 0] ; X)\}$, because if $\phi$ is a continuous function defined on $[-\rho, 0]$, $\lim _{s \rightarrow-\rho} \phi(s)$ exists automatically. The additional condition only makes sense for unbounded delay.

(iii) Indeed, for the unbounded delay case $\rho=\infty$, we have

$$
\mathbf{C}_{\infty}(X):=C((-\infty, 0] ; X)=\left\{\phi \in C((-\infty, 0] ; X) ; \lim _{s \rightarrow-\infty} \phi(s) \text { exists in } X\right\},
$$

which is a Banach space equipped with the norm

$$
\|\phi\|_{\mathbf{C}_{\infty}(X)}=\sup _{\theta \in(-\infty, 0]}\|\phi(\theta)\|_{X}
$$

Throughout this paper, we make use of several notations which are introduced in what follows. Let $\Omega$ be an open bounded set of $\mathbb{R}^{N},(\cdot, \cdot)$ and $|\cdot|$ denote the $L^{2}$-inner product and $L^{2}$-norm, $((\cdot, \cdot))$ and $\|\cdot\|$ denote the $H_{0}^{1}$-inner product and $H_{0}^{1}$-norm. Recall that for every $v \in H_{0}^{1}(\Omega)$, the Poincaré inequality

$$
\lambda_{1}(\Omega)|v|^{2} \leq\|v\|^{2}
$$


holds, where $\lambda_{1}(\Omega)$ is related to the domain $\Omega$ and is the first eigenvalue of $-\Delta$ with the zero Dirichlet boundary conditions. In the sequel, unless otherwise specified, we write $\lambda_{1}$ instead of $\lambda_{1}(\Omega)$ for simplicity.

From now on, we identify $L^{2}(\Omega)$ with its dual. Therefore, we have the usual chain of dense and compact embeddings $H_{0}^{1}(\Omega) \subset L^{2}(\Omega) \subset H^{-1}(\Omega)$. Thanks to the previous identification, $l(u)$ is in fact $(l, u)$. However, we keep the usual notation in the existing previous literature $l(u)$ instead of $(l, u)$ for the operator $l$ acting on $u$.

Now we will show the definitions of solutions to problem (2.1).

Definition 2.2 A weak solution to (2.1), corresponding to the initial value $\varphi \in \mathbf{C}_{\rho}\left(L^{2}(\Omega)\right)$, is a function $u \in L^{2}\left((\tau, T] ; H_{0}^{1}(\Omega)\right) \cap L^{\infty}\left((\tau, T] ; L^{2}(\Omega)\right)$ for all $T>\tau$, such that $u(t)=\varphi(t-\tau)$ for $t \in(\tau-\rho, \tau]$, and

$$
\frac{d}{d t}(u(t), v)+a(l(u(t)))((u(t), v))=(f(u(t)), v)+<h\left(t, u_{t}\right), v>, \quad \forall v \in H_{0}^{1}(\Omega),
$$

where the previous equation must be understood in the sense of $\mathcal{D}^{\prime}(\tau, \infty)$.

A notion of more regular solutions is also suitable.

Definition 2.3 A strong solution to (2.1) is a weak solution $u$ which also satisfies that $u \in$ $L^{2}((\tau, T] ; D(-\Delta)) \cap L^{\infty}\left((\tau, T] ; H_{0}^{1}(\Omega)\right)$ for all $T>\tau$.

To prove the existence of weak and strong solutions to problem (2.1), we will use the FaedoGalerkin approximations and pass to the limit by using compactness arguments. Thanks to spectral theory, there exists a sequence $\left\{w_{i}\right\}_{i \geq 1}$, which is a Hilbert basis of $L^{2}(\Omega)$ consisting of the eigenfunctions of $-\Delta$ in $H_{0}^{1}(\Omega)$.

Firstly, we consider the function $u^{n}(t):=\sum_{j=1}^{n} \gamma^{n j}(t) w_{j}$, for all $n \geq 1$,

$$
\begin{cases}\frac{d}{d t}\left(u^{n}(t), w_{j}\right)+a\left(l\left(u^{n}(t)\right)\right)\left(\left(u^{n}(t), w_{j}\right)\right)=\left(f\left(u^{n}(t)\right), w_{j}\right)+<h\left(t, u_{t}^{n}\right), w_{j}>, & t \in(\tau, \infty), \\ \left(u^{n}(\tau+\theta), w_{j}\right)=\left(\varphi(\theta), w_{j}\right) & \theta \in(-\rho, 0],\end{cases}
$$

where $j=1,2, \cdots, n$. Observe that (2.7) is a Cauchy problem to the following ordinary differential system in $\mathbb{R}^{n}$,

$$
\frac{d \gamma^{n j}(t)}{d t}+\lambda_{j} a\left(l\left(u^{n}(t)\right)\right) \gamma^{n j}(t)=\left(f\left(u^{n}(t)\right), w_{j}\right)+<h\left(t, u_{t}^{n}\right), w_{j}>, \quad j=1,2, \cdots, n,
$$

where $t \geq \tau, \lambda_{j}$ is the eigenvalue associated to the eigenfunction $w_{j}$ and the vector $\left(\gamma_{n 1}, \cdots, \gamma_{n n}\right)$ is unknown.

Proposition 2.4 Suppose that $a \in C\left(\mathbb{R} ; \mathbb{R}_{+}\right)$fulfils (2.2), $f(\cdot) \in C(\mathbb{R})$ verifies (2.3), $l \in L^{2}(\Omega)$, $h(\cdot, \cdot)$ satisfies conditions $\left(H_{1}\right)-\left(H_{4}\right)$, with $X=H_{0}^{1}(\Omega)$ and $Y=H^{-1}(\Omega)$, for an initial time $\tau \in \mathbb{R}$. 
Then for each initial value $\varphi \in \mathbf{C}_{\rho}\left(L^{2}(\Omega)\right)$, there exists at least one local solution $\left(\gamma_{n 1}, \cdots, \gamma_{n n}\right)$ of the ordinary differential system (2.8) defined on some interval $\left(\tau, t_{n}\right]$. Furthermore, if the function a is locally Lipschitz, f satisfies (2.4), and

$$
m^{2} \geq 4 C_{h}
$$

the uniqueness of local solution to problem (2.8) is guaranteed.

Proof. We split the proof into several steps.

Step 1: Existence of local solution. To do this, we are going to use the method of [18, Section 2.6], which is a generalization of Peano's Theorem to delay problem. Define

$$
\begin{aligned}
g: \quad & \rightarrow \mathbb{R}^{n} \\
& (t, \xi) \longmapsto\left(-\lambda_{1} z(\xi(0)) \xi^{1}(0)+\left(f\left(\sum_{i=1}^{n} \xi^{i}(0) w_{i}\right), w_{1}\right)+<h\left(t, \sum_{i=1}^{n} \xi^{i} w_{i}\right), w_{1}>+\cdots,\right. \\
& \left.-\lambda_{n} z(\xi(0)) \xi^{n}(0)+\left(f\left(\sum_{i=1}^{n} \xi^{i}(0) w_{i}\right), w_{n}\right)+<h\left(t, \sum_{i=1}^{n} \xi^{i} w_{i}\right), w_{n}>\right),
\end{aligned}
$$

where

$$
\Theta=\left\{(t, \xi) \in(\tau, T] \times \mathbf{C}_{\rho}\left(\mathbb{R}^{n}\right):\left|\xi-\left(\left(\varphi, w_{1}\right), \cdots,\left(\varphi, w_{n}\right)\right)\right| \leq b\right\},
$$

for any fixed $b \in \mathbb{R}^{+}$and

$$
\xi=\left(\xi^{1}, \cdots, \xi^{n}\right) \longmapsto z(\xi(0))=a\left(l\left(\sum_{i=1}^{n} \xi^{i}(0) w_{i}\right)\right) .
$$

Firstly, we are going to prove that $g$ is a Caratheodory function. Consider $\xi \in \mathbf{C}_{\rho}\left(\mathbb{R}^{n}\right)$ fixed, the function $g(\cdot, \xi)$ is measurable, because

$$
g_{j}(\cdot, \xi)=-\lambda_{j} z(\xi(0)) \xi^{j}(0)+f\left(\left(\sum_{i=1}^{n} \xi^{i}(0) w_{i}\right), w_{j}\right)+<h\left(t, \sum_{i=1}^{n} \xi^{i} w_{i}\right), w_{j}>
$$

is a measurable function as a consequence of Fubini's Theorem and condition $\left(H_{1}\right)$.

Secondly, we need to check that the function $g(t, \cdot)$ is continuous a.e. $t \in(\tau, T]$. Indeed,

$$
g_{j}(t, \xi)=-\lambda_{j} z(\xi(0)) \xi^{j}(0)+\left(f\left(\sum_{i=1}^{n} \xi^{i}(0) w_{i}\right), w_{j}\right)+<h\left(t, \sum_{i=1}^{n} \xi^{i} w_{i}\right), w_{j}>
$$

is a continuous function with respect to the second variable, because the functions $z(\xi(0)), \xi(0) \in$ $\mathbb{R}^{n} \rightarrow\left(f\left(\sum_{i=1}^{n} \xi^{i}(0) w_{i}\right), w_{j}\right)$, and $\xi \in \mathbf{C}_{\rho}\left(\mathbb{R}^{n}\right) \rightarrow<h\left(t, \sum_{i=1}^{n} \xi^{i} w_{i}\right), w_{j}>$ are continuous.

Thirdly, we are about to prove for each $(t, \xi) \in \Theta$, there exists a Lebesgue measurable function $m(\cdot) \in L^{1}(\tau, T)$ such that

$$
|g(\bar{t}, \bar{\xi})| \leq m(t), \quad \forall(\bar{t}, \bar{\xi}) \in V(t, \xi)
$$


where $V(t, \xi) \subset \Theta$ is a neighborhood of $(t, \xi)$. From the definition of $\Theta$, there exists a constant $C_{\Theta}$ (depending on $\varphi$ ) such that

$$
|\xi| \leq b+\left|\left(\left(\varphi, w_{1}\right), \cdots,\left(\varphi, w_{n}\right)\right)\right| \leq C_{\Theta}
$$

Next (2.3) implies that

$$
\left(f\left(\sum_{i=1}^{n} \xi^{i}(0) w_{i}\right), w_{j}\right) \leq 2^{\frac{1}{2}} C_{f}|\Omega|^{\frac{1}{2}}\left|w_{j}\right|+2^{\frac{1}{2}} C_{f} C_{\Theta}\left(\sum_{i=1}^{n}\left|\xi^{i}(0)\right|\right)\left|w_{j}\right| .
$$

At last, applying conditions $\left(H_{2}\right)-\left(H_{3}\right)$, we obtain

$$
\begin{aligned}
<h\left(t, \sum_{i=1}^{n} \xi^{i} w_{i}\right), w_{j}> & =<\left(h\left(t, \sum_{i=1}^{n} \xi^{i} w_{i}\right)-h(t, 0)\right), w_{j}> \\
& \leq\left\|h\left(t, \sum_{i=1}^{n} \xi^{i} w_{i}\right)-h(t, 0)\right\|_{\left.H^{-1}(\Omega)\right)}\left\|w_{j}\right\| \\
& \leq L_{h}\left\|\sum_{i=1}^{n} \xi^{i} w_{i}\right\|_{\mathbf{C}_{\rho}\left(H_{0}^{1}(\Omega)\right)}\left\|w_{j}\right\| \\
& \leq L_{h}\|\xi\|_{\mathbf{C}_{\rho}\left(\mathbb{R}^{n}\right)}\left(\sum_{i=1}^{n}\left\|w_{i}\right\|^{2}\right)^{1 / 2}\left\|w_{j}\right\| \\
& \leq L_{h} C_{\Theta}\left(\sum_{i=1}^{n}\left\|w_{i}\right\|^{2}\right)^{1 / 2}\left\|w_{j}\right\| .
\end{aligned}
$$

Taking into account (2.10) and owing to the continuity of the function $a$ in the compact interval $I^{\prime}:=\left[-|l| C_{\Theta} \sum_{i=1}^{n}\left|w_{i}\right|,|l| C_{\Theta} \sum_{i=1}^{n}\left|w_{i}\right|\right]$, there exists a constant $M>0$ such that

$$
z(\xi) \leq M, \quad \forall \xi \in \mathbf{C}_{\rho}\left(\mathbb{R}^{n}\right), \quad|\xi| \leq C_{\Theta} .
$$

Bearing this in mind, we obtain

$$
\begin{aligned}
\left|g_{j}(t, \xi)\right| \leq & \lambda_{j} z(\xi(0))\left|\xi^{j}(0)\right|+\left|\left(f\left(\sum_{i=1}^{n} \xi^{i}(0) w_{i}\right), w_{j}\right)\right|+\left|<h\left(t, \sum_{i=1}^{n} \xi^{i} w_{i}\right), w_{j}>\right| \\
\leq & \lambda_{j} M C_{\Theta}+2^{\frac{1}{2}} C_{f}|\Omega|^{\frac{1}{2}}\left|w_{j}\right|+2^{\frac{1}{2}} C_{f} C_{\Theta}\left(\sum_{i=1}^{n}\left|\xi^{i}(0)\right|\right)\left|w_{j}\right| \\
& +L_{h} C_{\Theta}\left(\sum_{i=1}^{n}\left\|w_{i}\right\|^{2}\right)^{1 / 2}\left\|w_{j}\right\| \in L^{1}(\tau, T) .
\end{aligned}
$$

In conclusion, there exists a local solution to problem (2.8).

Step 2: Uniqueness of local solution. Since the function $a$ is locally Lipschitz, for any bounded interval $[-R, R]$ of $\mathbb{R}$, there exists a positive constant $L_{a}(R)$ such that

$$
|a(x)-a(y)| \leq L_{a}(R)|x-y|, \quad \forall x, y \in[-R, R] .
$$


Assume that there exist two solutions $\varpi^{n}(t), \vartheta^{n}(t)$ of the ordinary differential system (2.8) in $\left(\tau, t_{1}\right]$ and $\left(\tau, t_{2}\right]$ respectively. Then it holds

$$
\begin{cases}\left(\varpi^{n j}(t)-\vartheta^{n j}(t)\right)^{\prime}=g_{j}\left(t, \varpi_{t}^{n}\right)-g_{j}\left(t, \vartheta_{t}^{n}\right), & t \in\left(\tau, \min \left\{t_{1}, t_{2}\right\}\right], \\ \left(\varpi^{n j}-\vartheta^{n j}\right)(t)=0, & t \in(\tau-\rho, \tau],\end{cases}
$$

where

$$
\begin{gathered}
g_{j}\left(t, \varpi_{t}^{n}\right)=-\lambda_{j}\left(a\left(l\left(u^{n}(t)\right)\right)\right) \varpi^{n j}(t)+\left(f\left(u^{n}(t)\right), w_{j}\right)+<h\left(t, u_{t}^{n}\right), w_{j}>, \\
g_{j}\left(t, \vartheta_{t}^{n}\right)=-\lambda_{j}\left(a\left(l\left(v^{n}(t)\right)\right)\right) \vartheta^{n j}(t)+\left(f\left(v^{n}(t)\right), w_{j}\right)+<h\left(t, v_{t}^{n}\right), w_{j}>,
\end{gathered}
$$

$u^{n}(t):=\sum_{j=1}^{n} \varpi^{n j}(t) w_{j}$ and $v^{n}(t):=\sum_{j=1}^{n} \vartheta^{n j}(t) w_{j}$.

Multiplying (2.12) by $\varpi^{n j}-\vartheta^{n j}$, summing from $j=1$ to $n$ and making use of (2.4), we obtain,

$$
\begin{aligned}
& \frac{1}{2} \frac{d}{d t}\left|u^{n}(t)-v^{n}(t)\right|^{2}+a\left(l\left(u^{n}(t)\right)\right)\left\|u^{n}(t)-v^{n}(t)\right\|^{2} \\
\leq & \left|a\left(l\left(u^{n}(t)\right)\right)-a\left(l\left(v^{n}(t)\right)\right)\right|\left|\left(\left(u^{n}(t), u^{n}(t)-v^{n}(t)\right)\right)\right|+\eta\left|u^{n}(t)-v^{n}(t)\right|^{2} \\
+ & <\left(h\left(t, u_{t}^{n}\right)-h\left(t, v_{t}^{n}\right)\right), u^{n}(t)-v^{n}(t)>.
\end{aligned}
$$

It is straightforward to deduce from $(2.7)$ that $u^{n}, v^{n} \in C\left(\left(\tau, \min \left\{t_{1}, t_{2}\right\}\right] ; L^{2}(\Omega)\right)$. When $\rho>0$ is a constant, obviously, there exist a constant $M^{\prime}>0$ and some $R>0$, such that

$$
\left|u^{n}(t)\right|^{2} \leq M^{\prime} \text { and }\left|v^{n}(t)\right|^{2} \leq M^{\prime}, \quad \forall t \in\left(\tau, \min \left\{t_{1}, t_{2}\right\}\right] .
$$

When $\rho=\infty$, by definition of the phase space $\mathbf{C}_{\infty}\left(L^{2}(\Omega)\right)$, the limit of $u^{n}(t)$ and $v^{n}(t)$ exist as $t$ goes to minus infinity, (2.13) also holds. In addition, taking into account that $l \in L^{2}(\Omega)$, it satisfies $\left\{l\left(u^{n}(t)\right)\right\}_{t \in\left(\tau, \min \left\{t_{1}, t_{2}\right\}\right]} \in[-R, R],\left\{l\left(v^{n}(t)\right)\right\}_{t \in\left(\tau, \min \left\{t_{1}, t_{2}\right\}\right]} \in[-R, R]$. Hence, using (2.2), (2.4), $\left(H_{4}\right)$ and the fact that function $a$ is locally Lipschitz (with Lipschitz constant $L_{a}(\cdot)$, cf. (2.11)), integrating the above inequality between $\tau$ and $t$, we deduce that

$$
\begin{gathered}
\left|u^{n}(t)-v^{n}(t)\right|^{2}+\frac{m}{2} \int_{\tau}^{t}\left\|u^{n}(s)-v^{n}(s)\right\|^{2} d s \\
\leq\left|u^{n}(\tau)-v^{n}(\tau)\right|^{2}+\left(\frac{\left(L_{a}(R)\right)^{2}|l|^{2} \lambda_{n}\left(M^{\prime}\right)^{2}}{m}+2 \eta\right) \int_{\tau}^{t}\left|u^{n}(s)-v^{n}(s)\right|^{2} d s \\
\quad+\frac{2 C_{h}}{m} \int_{\tau}^{t}\left\|u^{n}(s)-v^{n}(s)\right\|^{2} d s .
\end{gathered}
$$

With the help of (2.9), we have

$$
\left|u^{n}(t)-v^{n}(t)\right|^{2} \leq\left|u^{n}(\tau)-v^{n}(\tau)\right|^{2} e^{C t},
$$

where

$$
C=\frac{\left(L_{a}(R)\right)^{2}|l|^{2} \lambda_{n}\left(M^{\prime}\right)^{2}+2 m \eta}{m} .
$$

The proof of this proposition is finished. 
Remark 2.5 Condition (2.9), i.e., $m^{2} \geq 4 C_{h}$ can be read as: the combination of large effects of the nonlocal term and small delay source ensure the existence and uniqueness of solutions to ODE system (2.8). In fact, this condition also guarantees the existence and uniqueness of weak solutions to problem (2.1), all the details will be given in the next theorem.

Now we will show the existence and uniqueness of weak solutions and the continuity of the solutions in $L^{2}(\Omega)$ with respect to the initial value. Before going further, we need an extra condition:

$\left(H_{5}\right)$ If $v^{m}$ converges weakly to $v$ in $L^{2}\left((\tau-\rho, T] ; H_{0}^{1}(\Omega)\right)$ and strongly in $L^{2}\left((\tau-\rho, T] ; L^{2}(\Omega)\right)$, then $h\left(\cdot, v^{m}\right)$ converges weakly to $h(\cdot, v$. $)$ in $L^{2}\left((\tau, T] ; H^{-1}(\Omega)\right)$.

One example where our theory can be applied is as follows.

Example 2.6 Let $k \in L^{\infty}\left((-\rho, T] ; \mathcal{L}\left(H_{0}^{1}(\Omega) ; H^{-1}(\Omega)\right)\right)(\rho \geq \infty)$, and consider in problem (2.1), a term of the form

$$
h\left(t, u_{t}\right)=\int_{-\rho}^{0} k(t+s) u(t+s) d s,
$$

defined for all $u \in L^{2}\left((-\rho, T] ; H_{0}^{1}(\Omega)\right)$. This term corresponds to the situation

$$
h(t, \xi)=\int_{-\rho}^{0} k(t+s) \xi(s) d s
$$

for each $t \in[0, T]$ and $\xi \in \mathbf{C}_{\rho}\left(H_{0}^{1}(\Omega)\right)$. In this case, it is easy to see that $h$ is well defined and satisfies $\left(H_{1}\right)-\left(H_{5}\right)$.

Theorem 2.7 Suppose that a is locally Lipschitz and (2.2) is fulfilled, $f(\cdot) \in C(\mathbb{R})$ satisfies (2.3)(2.4), $l \in L^{2}(\Omega)$ and $h(\cdot, \cdot)$ satisfies conditions $\left(H_{1}\right)-\left(H_{5}\right)$, with $X=H_{0}^{1}(\Omega)$ and $Y=H^{-1}(\Omega)$. Also suppose (2.9) holds. Then, for each initial function $\varphi \in L^{2}\left((-\rho, 0] ; H_{0}^{1}(\Omega)\right) \cap \mathbf{C}_{\rho}\left(L^{2}(\Omega)\right)$, there exists a weak solution to problem (2.1). In addition, this solution is continuous in $L^{2}(\Omega)$ with respect to the initial data.

Proof. We split the proof into several steps.

Step 1: Existence of weak solution. Multiplying by $\gamma^{n j}$ in (2.8), summing from $j=1$ to $n$ and using (2.2), we obtain

$$
\frac{d}{d t}\left|u^{n}(t)\right|^{2}+2 m\left\|u^{n}(t)\right\|^{2} \leq 2\left(f\left(u^{n}(t)\right), u^{n}(t)\right)+2<h\left(t, u_{t}^{n}\right), u^{n}(t)>, \quad \text { a.e. } t \in\left(\tau, t_{n}\right),
$$

where $\left(\tau, t_{n}\right)$ is the interval of existence of maximal solution. By the Young inequality and (2.3), for a.e. $t \in\left(\tau, t_{n}\right)$, we have

$$
\frac{d}{d t}\left|u^{n}(t)\right|^{2}+m\left\|u^{n}(t)\right\|^{2} \leq \frac{4 C_{f}^{2}}{\lambda_{1} m}|\Omega|+\frac{4 C_{f}^{2}}{\lambda_{1} m}\left|u^{n}(t)\right|^{2}+\frac{2}{m}\left\|h\left(t, u_{t}^{n}\right)\right\|_{H^{-1}(\Omega)}^{2} .
$$


Integrating above inequality between $\tau$ and $t \in\left(\tau, t_{n}\right)$, together with condition $\left(H_{4}\right)$, we obtain

$$
\begin{aligned}
\left|u^{n}(t)\right|^{2}+m \int_{\tau}^{t}\left\|u^{n}(s)\right\|^{2} d s \leq & \left|u^{n}(\tau)\right|^{2}+\frac{4 C_{f}^{2}|\Omega|(T-\tau)}{\lambda_{1} m}+\frac{4 C_{f}^{2}}{\lambda_{1} m} \int_{\tau}^{t}\left|u^{n}(s)\right|^{2} d s \\
& +\frac{2 C_{h}}{m}\|\varphi\|_{L^{2}\left((-\rho, 0] ; H_{0}^{1}(\Omega)\right)}^{2}+\frac{2 C_{h}}{m} \int_{\tau}^{t}\left\|u^{n}(s)\right\|^{2} d s .
\end{aligned}
$$

The Gronwall lemma and (2.9) imply for each $n \in \mathbb{N}, u^{n}(\cdot)$ is well defined on $(\tau, t]$ and $u^{n}(t)$ is bounded for all $t \in\left(\tau, t_{n}\right)$. Therefore, it is straightforward to check that $\left\{u^{n}\right\}$ is well defined on $(\tau, T]$ for all $n \in \mathbb{N}^{+}$. Actually, $\left\{u^{n}\right\}$ is bounded in $L^{\infty}\left((\tau, T] ; L^{2}(\Omega)\right) \cap L^{2}\left((\tau, T] ; H_{0}^{1}(\Omega)\right)$. Moreover, notice that, for each $n \in \mathbb{N}, u^{n} \in C\left((\tau, T] ; L^{2}(\Omega)\right)$, we deduce there exists a positive constant $C$ independent of $n$, such that

$$
\left|u^{n}(t)\right| \leq C, \quad \forall t \in(\tau, T], \quad \forall n \geq 1 .
$$

Combining with $a \in C\left(\mathbb{R} ; \mathbb{R}^{+}\right)$and $l \in L^{2}(\Omega)$, there exists a positive constant $M_{C}$ such that

$$
a\left(l\left(u^{n}(t)\right)\right) \leq M_{C}, \quad \forall t \in(\tau, T], \quad \forall n \geq 1 .
$$

Hence,

$$
\int_{\tau}^{T}\left|a\left(l\left(u^{n}(t)\right)\right)\right|^{2}\left\|-\Delta u^{n}(t)\right\|_{H^{-1}(\Omega)}^{2} d t \leq\left(M_{C}\right)^{2} \int_{\tau}^{T}\left\|u^{n}(t)\right\|^{2} d t .
$$

Taking into account that $\left\{u^{n}\right\}$ is bounded in $L^{2}\left((\tau, T] ; H_{0}^{1}(\Omega)\right)$, we deduce that the sequence $\left\{-a\left(l\left(u^{n}\right)\right) \Delta u^{n}\right\}$ is bounded in $L^{2}\left((\tau, T] ; H^{-1}(\Omega)\right)$.

On the other hand, using (2.3), we have

$$
\begin{aligned}
\int_{\tau}^{T} \int_{\Omega}\left|f\left(u^{n}(t, x)\right)\right|^{2} d x d t & \leq 2 C_{f}^{2} \int_{\tau}^{T} \int_{\Omega}\left(1+\left|u^{n}(x, t)\right|^{2}\right) d x d t \\
& \leq 2 C_{f}^{2}|\Omega|(T-\tau)+2 C_{f}^{2} \int_{\tau}^{T}\left|u^{n}(t)\right|^{2} d t
\end{aligned}
$$

Since $\left\{u^{n}\right\}$ is bounded in $L^{\infty}\left((\tau, T] ; L^{2}(\Omega)\right),\left\{f\left(u^{n}\right)\right\}$ is bounded in $L^{2}\left((\tau, T] ; L^{2}(\Omega)\right)$ holds immediately.

In addition, to prove $u^{n}$ converges to $u$ strongly in $L^{2}\left((\tau, T] ; L^{2}(\Omega)\right)$, we need to state the sequence $\left\{\left(u^{n}\right)^{\prime}\right\}$ is bounded in $L^{2}\left((\tau, T] ; H^{-1}(\Omega)\right)$. To this end, let us define the following projectors,

$$
\begin{aligned}
\tilde{P}_{n}: & H^{-1}(\Omega) \longrightarrow H^{-1}(\Omega) \\
& f \longrightarrow\left[\phi \in H_{0}^{1}(\Omega) \longrightarrow<\tilde{P}_{n} f, \phi>:=<f, P_{n} \phi>\right]
\end{aligned}
$$

where

$$
\begin{aligned}
P_{n}: & L^{2}(\Omega) \longrightarrow V_{n}:=\operatorname{span}\left[w_{1}, \cdots, w_{n}\right] \\
& \phi \longrightarrow \sum_{j=1}^{n}\left(\phi, w_{j}\right) w_{j} .
\end{aligned}
$$


Observe that $\tilde{P}_{n}$ is the continuous extension in $H^{-1}(\Omega)$ of $P_{n}$. Then, in what follows, we will make an abuse of notation and denote this projection by $P_{n}$.

Bearing this in mind, together with $(2.14)-(2.15),\left(H_{4}\right)$ and the definition of the above projectors, we have

$$
\begin{aligned}
& \int_{\tau}^{T}\left\|\frac{d u^{n}(t)}{d t}\right\|_{H^{-1}(\Omega)}^{2} d t \\
& \quad=\int_{\tau}^{T}\left\|a\left(l\left(u^{n}(t)\right)\right) \Delta u^{n}(t)+P_{n} f\left(u^{n}(t)\right)+P_{n} h\left(t, u_{t}^{n}\right)\right\|_{H^{-1}(\Omega)}^{2} d t \\
& \quad \leq 3\left(M_{C}\right)^{2} \int_{\tau}^{T}\left\|u^{n}(t)\right\|^{2} d t+\frac{3}{\lambda_{1}} \int_{\tau}^{T}\left|P_{n}\left(f\left(u^{n}(t)\right)\right)\right|^{2} d t+3 \int_{\tau}^{T}\left\|P_{n} h\left(t, u_{t}^{n}\right)\right\|_{H^{-1}(\Omega)}^{2} d t \\
& \quad \leq\left(3\left(M_{C}\right)^{2}+\frac{6 C_{f}^{2}}{\lambda_{1}^{2}}+3 C_{h}\right) \int_{\tau}^{T}\left\|u^{n}(t)\right\|^{2} d t+\frac{6 C_{f}^{2}|\Omega|}{\lambda_{1}}(T-\tau)+3 C_{h}\left\|\varphi^{n}(t)\right\|_{L^{2}\left((-\rho, 0] ; H_{0}^{1}(\Omega)\right)}^{2},
\end{aligned}
$$

namely, the sequence $\left\{\left(u^{n}\right)^{\prime}\right\}$ is bounded in $L^{2}\left((\tau, T] ; H^{-1}(\Omega)\right)$.

Therefore, compactness arguments and the Aubin-Lions lemma imply there exists a subsequence of $\left\{u^{n}\right\}$ (relabeled the same) and $u \in L^{\infty}\left((\tau, T] ; L^{2}(\Omega)\right) \cap L^{2}\left((\tau, T] ; H_{0}^{1}(\Omega)\right)$ with $\left\{\left(u^{n}\right)^{\prime}\right\} \in$ $L^{2}\left((\tau, T] ; H^{-1}(\Omega)\right)$, such that

$$
\left\{\begin{array}{l}
u^{n} \rightarrow u \text { weakly-star in } L^{\infty}\left((\tau, T] ; L^{2}(\Omega)\right), \\
u^{n} \rightarrow u \text { weakly in } L^{2}\left((\tau, T] ; H_{0}^{1}(\Omega)\right), \\
\left(u^{n}\right)^{\prime} \rightarrow u^{\prime} \text { weakly in } L^{2}\left((\tau, T] ; H^{-1}(\Omega)\right), \\
u^{n} \rightarrow u \text { strongly in } L^{2}\left((\tau, T] ; L^{2}(\Omega)\right), \\
u^{n}(x, t) \rightarrow u(x, t) \text { a.e. }(x, t) \in \Omega \times(\tau, T], \\
u^{n}(t) \rightarrow u(t) \text { strongly in } L^{2}(\Omega), \text { a.e. } t \in(\tau, T], \\
f\left(u^{n}(t)\right) \rightarrow \xi_{1} \text { weakly in } L^{2}\left((\tau, T] ; L^{2}(\Omega)\right), \\
a\left(l\left(u^{n}\right)\right) u^{n} \rightarrow \xi_{2} \text { weakly in } L^{2}\left((\tau, T] ; H_{0}^{1}(\Omega)\right),
\end{array}\right.
$$

for all $T>\tau$.

In the end, we pass to the limit for each term to prove the existence of weak solutions to problem (2.1). Firstly, by $(2.16)$ and $\left(H_{5}\right)$, it follows

$$
h\left(t, u_{t}^{n}\right) \rightarrow h\left(t, u_{t}\right) \quad \text { weakly in } L^{2}\left((\tau, T] ; H^{-1}(\Omega)\right) .
$$

Secondly, we need to check that $\xi_{1}=f(u)$. Since $u^{n}(\cdot)$ converges to $u(\cdot)$ strongly in $L^{2}(\Omega)$, a.e. $t \in(\tau, T]$, we deduce that

$$
\begin{gathered}
u^{n}(x, t) \rightarrow u(x, t), \quad \forall(x, t) \in \Omega \times(\tau, T] \backslash N_{1}, \\
u^{n}(t) \rightarrow u(t) \quad \text { strongly in } \quad L^{2}(\Omega), \quad \forall t \in(\tau, T] \backslash N_{2},
\end{gathered}
$$


where $N_{1}$ is a null set in $\mathbb{R}^{N+1}$ and $N_{2}$ is a null set in $\mathbb{R}$. From this, together with the fact that $f \in C(\mathbb{R}),\left\{f\left(u^{n}\right)\right\}$ is bounded in $L^{2}\left((\tau, T] ; L^{2}(\Omega)\right)$ and converges pointwisely to $f(u)$ a.e. $\Omega \times(\tau, T)$ (cf. (2.18)), by applying [22, Lemma 1.3] it follows $\xi_{1}=f(u)$.

Finally, we will prove that $\xi_{2}=a(l(u)) u$. As $a \in C\left(\mathbb{R} ; \mathbb{R}_{+}\right), l \in L^{2}(\Omega)$ and (2.19) holds, we have

$$
a\left(l\left(u^{n}(t)\right)\right) \rightarrow a(l(u(t))), \quad \forall t \in(\tau, T] \backslash N_{2} .
$$

Therefore,

$$
a\left(l\left(u^{n}(t)\right)\right) u^{n}(x, t) \rightarrow a(l(u(t))) u(x, t), \quad \forall(x, t) \in \Omega \times(\tau, T] \backslash\left(N_{1} \cup\left(\Omega \times N_{2}\right)\right),
$$

where $N_{1} \cup\left(\Omega \times N_{2}\right)$ is a null set in $\mathbb{R}^{N+1}$. $\xi_{2}=a(l(u)) u$ follows immediately again by applying [22, Lemma 1.3], since $\left\{a\left(l\left(u^{n}\right)\right) u^{n}\right\}$ is bounded in $L^{2}\left((\tau, T] ; H_{0}^{1}(\Omega)\right)$.

Now, passing to the limit in (2.7), taking into account (2.16) and the fact that $\cup_{n \in \mathbb{N}} V_{n}$ is dense in $H_{0}^{1}(\Omega),(2.6)$ holds for all $v \in H_{0}^{1}(\Omega)$. Thus, till now, we have proved that $u$ is a weak solution to problem (2.1) for $t \in(\tau, T]$. Besides, when $t \in(\tau-\rho, \tau)$, from the definition of projection $P_{n}$, we have

$$
u^{n}(t)=P_{n} \varphi(t-\tau) \rightarrow \varphi(t-\tau)=u(t) \quad \text { as } \quad n \rightarrow \infty .
$$

Therefore, to prove that $u \in C\left((\tau-\rho, T] ; L^{2}(\Omega)\right)$ is a weak solution to $(2.1)$, we only need to check $u(\tau)=\varphi(0)$.

On the one hand, consider fixed $n, \phi \in H^{1}(\tau, T]$ with $\phi(T)=0$ and $\phi(\tau) \neq 0$, and $w \in V_{n}$. Multiplying by $\phi$ in (2.7), integrating between $\tau$ and $T$, we obtain for all $m>n$,

$$
\begin{aligned}
& -\left(\varphi^{m}(0), w\right) \phi(\tau)-\int_{\tau}^{T} \phi^{\prime}(t)\left(u^{m}(t), w\right) d t+\int_{\tau}^{T} a\left(l\left(u^{m}(t)\right)\right)<-\Delta u^{m}(t), w>\phi(t) d t \\
& =\int_{\tau}^{T}\left(f\left(u^{m}(t)\right), w\right) \phi(t) d t+\int_{\tau}^{T}<h\left(t, u_{t}^{m}\right), w>\phi(t) d t .
\end{aligned}
$$

Taking limit when $m \rightarrow \infty$ and using (2.16)-(2.17), we deduce

$$
\begin{aligned}
& -(\varphi(0), w) \phi(\tau)-\int_{\tau}^{T} \phi^{\prime}(t)(u(t), w) d t+\int_{\tau}^{T} a(l(u(t)))<-\Delta u(t), w>\phi(t) d t \\
& =\int_{\tau}^{T}(f(u(t)), w) \phi(t) d t+\int_{\tau}^{T}<h\left(t, u_{t}\right), w>\phi(t) d t .
\end{aligned}
$$

On the other hand, multiplying by $\phi$ in (2.6) and integrating between $\tau$ and $T$, we obtain

$$
\begin{aligned}
& -(u(\tau), w) \phi(\tau)-\int_{\tau}^{T} \phi^{\prime}(t)(u(t), w) d t+\int_{\tau}^{T} a(l(u(t)))<-\Delta u(t), w>\phi(t) d t \\
& =\int_{\tau}^{T}(f(u(t)), w) \phi(t) d t+\int_{\tau}^{T}<h\left(t, u_{t}\right), w>\phi(t) d t .
\end{aligned}
$$

Comparing (2.20) and the above expression, we have $(u(\tau), w) \phi(\tau)=(\varphi(0), w)) \phi(\tau)$. As $\phi(\tau) \neq 0$ and $\left\{w_{j}\right\}$ is a Hilbert basis of $L^{2}(\Omega)$, we conclude that $u(\tau)=\varphi(0)$. 
Step 2: Uniqueness of solution and continuity w.r.t. initial data. Assume there exist two weak solutions $u$ and $v$, with the initial functions $\varphi(\cdot)$ and $\nu(\cdot)$ respectively. From the energy equality, we obtain

$$
\begin{aligned}
& \frac{1}{2} \frac{d}{d t}|u(t)-v(t)|^{2}+a(l(u(t)))\|u(t)-v(t)\|^{2} \\
= & {[a(l(u(t)))-a(l(v(t)))]((u(t), u(t)-v(t)))+(f(u(t))-f(v(t)), u(t)-v(t)) } \\
+ & <h\left(t, u_{t}\right)-h\left(t, v_{t}\right), u(t)-v(t)>.
\end{aligned}
$$

Since $u, v \in C\left([\tau, T] ; L^{2}(\Omega)\right)$, there exists a bounded set $\mathcal{S} \subset L^{2}(\Omega)$ such that $\{z(t)\}_{t \in[\tau, T]} \subset \mathcal{S}$, where $z$ is either $u$ or $v$. Besides, taking into account that $l \in L^{2}(\Omega)$, there exists a constant $R>0$ such that $\{l(z(t))\}_{t \in[\tau, T]} \subset[-R, R]$. Then, by means of $(2.2),(2.4),\left(H_{3}\right)$ and the locally Lipschitz continuity of the function $a$ (cf. (2.11)), we obtain

$$
\begin{aligned}
& \frac{1}{2} \frac{d}{d t}|u(t)-v(t)|^{2}+m\|u(t)-v(t)\|^{2} \\
& \leq L_{a}(R)|l||u(t)-v(t)|\|v(t)\|\|u(t)-v(t)\|+\eta|u(t)-v(t)|^{2} \\
& +<h\left(t, u_{t}\right)-h\left(t, v_{t}\right), u(t)-v(t)>.
\end{aligned}
$$

Integrating (2.21) between $\tau$ and $t$, by $\left(H_{4}\right)$ and the Young inequality, we have

$$
\begin{aligned}
& |u(t)-v(t)|^{2}+\frac{m}{2} \int_{\tau}^{t}\|u(s)-v(s)\|^{2} d s \\
& \leq|\varphi(0)-\nu(0)|^{2}+\frac{\left(L_{a}(R)\right)^{2}|l|^{2}}{m} \int_{\tau}^{t}|u(s)-v(s)|^{2}\|v(s)\|^{2} d s \\
& \quad+\frac{2 C_{h}}{m}\left(\|\varphi-\nu\|_{L^{2}\left((-\rho, 0] ; H_{0}^{1}(\Omega)\right)}^{2}\right)+\frac{2 C_{h}}{m} \int_{\tau}^{t}\|u(s)-v(s)\|^{2} d s .
\end{aligned}
$$

Now, applying the Gronwall lemma and (2.9) to the above expression, we have

$$
\begin{aligned}
|u(t)-v(t)|^{2} \leq & \left(|\varphi(0)-\nu(0)|^{2}+\frac{2 C_{h}}{m}\|\varphi-\nu\|_{L^{2}\left((-\rho, 0] ; H_{0}^{1}(\Omega)\right)}^{2}\right) \\
& \times \exp \left\{\frac{\left(L_{a}(R)\right)^{2}|l|^{2}\|v\|_{L^{2}\left((\tau, T] ; H_{0}^{1}(\Omega)\right)}^{2}}{m}\right\}(t-\tau), \quad \forall t \in(\tau, T] .
\end{aligned}
$$

Hence, both results, the uniqueness of solutions and the continuity with respect to the initial data of problem (2.1), follow immediately.

Remark 2.8 If we assume $h(\cdot, \cdot): \mathbb{R} \times \mathbf{C}_{\rho}\left(L^{2}(\Omega)\right) \rightarrow L^{2}(\Omega)$ satisfies $\left(H_{1}\right)-\left(H_{4}\right)$ with $X=Y=$ $L^{2}(\Omega)$, then we are able to obtain the same result as in Theorem 2.7 without assuming condition (2.9). Moreover, the initial function $\varphi$ can be taken with less regularity, i.e., we can prove the result for $\varphi \in \mathbf{C}_{\rho}\left(L^{2}(\Omega)\right)$. 
In the following result, we will study the regularising effect of the equation. In addition, taking a more regular initial datum, the existence of a strong solution to problem (2.1) will be analyzed.

Theorem 2.9 Assume hypotheses of Theorem 2.7, where $h: \mathbb{R} \times \mathbf{C}_{\rho}\left(L^{2}(\Omega)\right) \rightarrow L^{2}(\Omega)$ satisfies $\left(H_{1}\right)-\left(H_{4}\right)$ with $X=Y=L^{2}(\Omega)$. Then, for every $\varepsilon>0$ and $T>\varepsilon+\tau$, the weak solution $u$ belongs to $C\left((\tau, T] ; H_{0}^{1}(\Omega)\right) \cap L^{2}((\varepsilon+\tau, T] ; D(-\Delta))$. In fact, if the initial function $\varphi \in L^{2}\left((-\rho, 0] ; H_{0}^{1}(\Omega)\right)$, then the function $u \in C\left((\tau, T] ; H_{0}^{1}(\Omega)\right) \cap L^{2}((\tau, T] ; D(-\Delta))$ for every $T>\tau$.

Proof. We split the proof into two steps.

Step 1: Regularising effect. Multiplying by $\lambda_{j} \gamma^{n j}$ in (2.7), summing from $j=1$ to $n$, and using (2.2), (2.4), the Cauchy and Poincaré inequalities, it yields

$$
\frac{d}{d t}\left\|u^{n}(t)\right\|^{2}+m\left|-\Delta u^{n}(t)\right|^{2} \leq \frac{4 C_{f}^{2}|\Omega|}{m}+\frac{4 C_{f}^{2}}{\lambda_{1} m}\left\|u^{n}(t)\right\|^{2}+\frac{2}{m}\left|h\left(t, u_{t}^{n}\right)-h(t, 0)\right|^{2}, \quad \text { a.e. } t \geq \tau \text {. }
$$

Integrating the above inequality between $s$ and $t$ with $\tau<s \leq t \leq T$, we obtain

$$
\begin{aligned}
& \left\|u^{n}(t)\right\|^{2}+m \int_{s}^{t}\left|-\Delta u^{n}(r)\right|^{2} d r \leq\left\|u^{n}(s)\right\|^{2}+\frac{4 C_{f}^{2}|\Omega|}{m}(T-\tau)+\frac{4 C_{f}^{2}}{\lambda_{1} m} \int_{s}^{t}\left\|u^{n}(r)\right\|^{2} d r \\
& +\frac{2}{m} \int_{s}^{t}\left|h\left(r, u_{r}^{n}\right)-h(r, 0)\right|^{2} d r .
\end{aligned}
$$

Now, integrating in (2.22) between $\tau$ and $t$, by $\left(H_{4}\right)$, we deduce that

$$
\begin{aligned}
(t-\tau)\left\|u^{n}(t)\right\|^{2} \leq & \frac{4 C_{f}^{2}|\Omega|(T-\tau)^{2}}{m}+\left(\frac{4 C_{f}^{2}(T-\tau)}{\lambda_{1} m}+1\right) \int_{\tau}^{t}\left\|u^{n}(r)\right\|^{2} d r \\
& +\frac{2(T-\tau)}{m} \int_{\tau}^{t}\left|h\left(r, u_{r}^{n}\right)-h(r, 0)\right|^{2} d r \\
\leq & \frac{4 C_{f}^{2}|\Omega|(T-\tau)^{2}}{m}+\left(\frac{4 C_{f}^{2}(T-\tau)+2 C_{h}(T-\tau)}{\lambda_{1} m}+1\right) \int_{\tau}^{t}\left\|u^{n}(r)\right\|^{2} d r \\
& +\frac{2 C_{h}(T-\tau)}{\lambda_{1} m}\|\varphi\|_{L^{2}\left((-\rho, 0] ; H_{0}^{1}(\Omega)\right)}^{2} .
\end{aligned}
$$

Therefore,

$$
\begin{aligned}
\left\|u^{n}(t)\right\|^{2} \leq & \frac{4 C_{f}^{2}|\Omega|(T-\tau)^{2}}{\varepsilon m}+\left(\frac{4 C_{f}^{2}(T-\tau)+2 C_{h}(T-\tau)+\lambda_{1} m}{\lambda_{1} m \varepsilon}\right) \\
& \times \int_{\tau}^{t}\left\|u^{n}(r)\right\|^{2} d r+\frac{2 C_{h}(T-\tau)}{\lambda_{1} m \varepsilon}\|\varphi\|_{L^{2}\left((-\rho, 0] ; H_{0}^{1}(\Omega)\right)}^{2},
\end{aligned}
$$

for all $t \in(\tau+\varepsilon, T]$ with $\varepsilon \in(0, T-\tau)$. 
From this and taking into account the boundedness of $\left\{u^{n}\right\}$ in $L^{2}\left((\tau, T] ; H_{0}^{1}(\Omega)\right)$, we deduce that $\left\{u^{n}\right\}$ is bounded in $L^{\infty}\left((\tau, T] ; H_{0}^{1}(\Omega)\right)$. As a byproduct, the boundedness of $\left\{u^{n}\right\}$ in $L^{2}((\tau+\varepsilon, T] ; D(-\Delta))$ holds true just taking $s=\tau$ and $t=T$ in (2.22). In addition, by means of this more regular boundedness, we deduce that the sequence $\left\{\left(u^{n}\right)^{\prime}\right\}$ is bounded in $L^{2}\left((\varepsilon+\tau, T] ; L^{2}(\Omega)\right)$. Thanks to the uniqueness of the weak solution, $\left\{u^{n}\right\}$ converges to $u$ weakly in $L^{2}((\tau+\varepsilon, T] ; D(-\Delta))$ and $\left\{\left(u^{n}\right)^{\prime}\right\}$ converges to $\left\{u^{\prime}\right\}$ weakly in $L^{2}\left((\tau+\varepsilon, T] ; L^{2}(\Omega)\right)$. As a consequence, $u \in L^{2}((\tau+\varepsilon, T] ; D(-\Delta)) \cap C\left((\tau, T] ; H_{0}^{1}(\Omega)\right)$.

Step 2: Strong solution. In this step, if $\varphi \in L^{2}\left((-\rho, 0] ; H_{0}^{1}(\Omega)\right)$, we will show that $u \in$ $L^{2}((\tau, T] ; D(-\Delta)) \cap C\left((\tau, T] ; H_{0}^{1}(\Omega)\right)$ for all $T>\tau$. To that end, we multiply $(2.7)$ by $\lambda_{j} \gamma^{n j}$, sum from $j=1$ to $n$ and use $(2.2)$, obtaining

$$
\frac{1}{2} \frac{d}{d t}\left\|u^{n}(t)\right\|^{2}+m\left|-\Delta u^{n}(t)\right|^{2} \leq\left(f\left(u^{n}(t)\right),-\Delta u^{n}(t)\right)+\left(h\left(t, u_{t}^{n}\right),-\Delta u^{n}(t)\right), \text { a.e. } t \in(\tau, T] .
$$

Now, from (2.3) and the Young inequality, we have

$$
\begin{aligned}
\left(f\left(u^{n}(t)\right),-\Delta u^{n}(t)\right) & \leq \frac{1}{m}\left|f\left(u^{n}(t)\right)\right|^{2}+\frac{m}{4}\left|-\Delta u^{n}(t)\right|^{2} \\
& \leq \frac{2 C_{f}^{2}|\Omega|}{m}+\frac{2 C_{f}^{2}}{m}\left|u^{n}(t)\right|^{2}+\frac{m}{4}\left|-\Delta u^{n}(t)\right|^{2},
\end{aligned}
$$

and by $\left(H_{2}\right)$,

$$
\left(h\left(t, u_{t}^{n}\right),-\Delta u^{n}(t)\right) \leq \frac{1}{m}\left|h\left(t, u_{t}^{n}\right)-h(t, 0)\right|^{2}+\frac{m}{4}\left|-\Delta u^{n}(t)\right|^{2} .
$$

Taking this into account, from (2.23), we deduce that

$$
\frac{d}{d t}\left\|u^{n}(t)\right\|^{2}+m\left|-\Delta u^{n}(t)\right|^{2} \leq \frac{4 C_{f}^{2}|\Omega|}{m}+\frac{4 C_{f}^{2}}{m \lambda_{1}}\left\|u^{n}(t)\right\|^{2}+\frac{2}{m}\left|h\left(t, u_{t}^{n}\right)-h(t, 0)\right|^{2}, \quad \text { a.e. } t>\tau \text {. }
$$

Integrating (2.24) between $\tau$ and $t, t \in(\tau, T]$, by $\left(H_{4}\right)$ we have

$$
\begin{aligned}
\left\|u^{n}(t)\right\|^{2}+ & m \int_{\tau}^{t}\left|-\Delta u^{n}(s)\right|^{2} d s \\
\leq & \|\varphi(0)\|^{2}+\frac{4 C_{f}^{2}|\Omega|(T-\tau)}{m}+\left(\frac{4 C_{f}^{2}+2 C_{h}}{m \lambda_{1}}\right) \int_{\tau}^{t}\left\|u^{n}(s)\right\|^{2} d s \\
& +\frac{2 C_{h}}{m \lambda_{1}}\|\varphi\|_{L^{2}\left((-\rho, 0] ; H_{0}^{1}(\Omega)\right)}^{2} .
\end{aligned}
$$

Since $\left\{u^{n}\right\}$ is bounded in $L^{2}\left((\tau, T] ; H_{0}^{1}(\Omega)\right)$, the sequence $\left\{u^{n}\right\}$ is bounded in $L^{\infty}\left((\tau, T] ; H_{0}^{1}(\Omega)\right)$ $\cap L^{2}((\tau, T] ; D(-\Delta))$. As a result, the sequence $\left\{\left(u^{n}\right)^{\prime}\right\}$ is bounded in $L^{2}\left((\tau, T] ; L^{2}(\Omega)\right)$. Thanks to the uniqueness of weak solution, $\left\{u^{n}\right\}$ converges to $u$ weakly-star in $L^{\infty}\left((\tau, T] ; H_{0}^{1}(\Omega)\right)$ and weakly in $L^{2}((\tau, T] ; D(-\Delta))$, also $\left\{\left(u^{n}\right)^{\prime}\right\}$ converges to $u^{\prime}$ weakly in $L^{2}\left((\tau, T] ; L^{2}(\Omega)\right)$. Therefore, we have $u \in C\left((\tau, T] ; H_{0}^{1}(\Omega)\right)$, since $u \in L^{\infty}\left((\tau, T] ; H_{0}^{1}(\Omega)\right) \cap L^{2}((\tau, T] ; D(-\Delta))$, and $u^{\prime} \in L^{2}\left((\tau, T] ; L^{2}(\Omega)\right)$ as well. The proof is finished. 


\section{Analysis of the stationary problem}

\subsection{Statement of the problem}

In this section, we are interested in studying the asymptotic behavior of the stationary (steady state) solutions of problem (2.1). Recall that a stationary (steady state) solution to our problem is an element $u^{*} \in H_{0}^{1}(\Omega)$, such that

$$
-a\left(l\left(u^{*}\right)\right) \Delta u^{*}=f\left(u^{*}\right)+h\left(t, u^{*}\right), \quad \forall t \in \mathbb{R} .
$$

To state this problem, we need to assume $h\left(t, u^{*}\right)$ is independent of $t$, i.e., there exists a mapping $H: H_{0}^{1}(\Omega) \rightarrow H^{-1}(\Omega)$ such that

$$
h(t, \xi)=H\left(\xi^{*}\right)
$$

whenever $\xi(\theta)=\xi^{*}$, for all $\theta \leq 0$.

For instance, if $H: \mathbb{R} \rightarrow \mathbb{R}$ is Lipschitz continuous and we define $h(t, \xi)=H(\xi(-\delta(t)))$ via the Nemytskii operator, for $\delta(\cdot): \mathbb{R}^{+} \rightarrow(0, \rho)$, i.e., $H(\xi(-\delta(t)))(x)=H(\xi(-\delta(t))(x))$, then $h\left(t, \xi^{*}\right)=H\left(\xi^{*}\right)$.

Another example satisfying our problem is the case of distributed delay: $h(t, \xi)=\int_{-\rho}^{0} k(s, \xi(s)) d s$ with $k: \mathbb{R}^{2} \rightarrow \mathbb{R}$ a Lipschitz continuous function with respect to its second variable. For more details, see [23].

This leads us to first analyze the following elliptic problem,

$$
\begin{cases}-a(l(u)) \Delta u=f(u)+H(u), & \text { in } \Omega, \\ u=0, & \text { on } \partial \Omega,\end{cases}
$$

where the functions $a$ and $f$ are globally Lipschitz, with respective Lipschitz constants $L_{a}, L_{f} \geq 0$ and there exist positive constants $m$ and $M$, such that

$$
0<m \leq a(s) \leq M, \quad \forall s \in \mathbb{R}
$$

\subsection{The existence and uniqueness of stationary solutions}

Definition 3.1 A weak solution to (3.1) is an element $u^{*} \in H_{0}^{1}(\Omega)$ such that

$$
a\left(l\left(u^{*}\right)\right)\left(\left(u^{*}, v\right)\right)=\left(f\left(u^{*}\right), v\right)+<H\left(u^{*}\right), v>, \quad \forall v \in H_{0}^{1}(\Omega) .
$$

In what follows, we analyze the existence of solutions to (3.1) by a corollary of the Brouwer fixed point theorem. The uniqueness as well as the stability is also studied under suitable assumptions.

Theorem 3.2 Let $a, f$ and $H: H_{0}^{1}(\Omega) \rightarrow H^{-1}(\Omega)$ be globally Lipschitz functions, with Lipschitz constants $L_{a}, L_{f}$ and $L_{H}$, respectively. Assume (3.2) is also satisfied, $l \in L^{2}(\Omega)$ and $m>$ $L_{f} \lambda_{1}^{-1}+L_{H}$. Then: 
1. There exists at least one solution to (3.1). In addition, any solution $u^{*}$ to (3.1) fulfills

$$
\left\|u^{*}\right\| \leq \Upsilon:=\frac{\lambda_{1}^{-1 / 2}|\Omega|^{1 / 2}|f(0)|+\|H(0)\|_{H^{-1}(\Omega)}}{m-\lambda_{1}^{-1} L_{f}-L_{H}} .
$$

2. If the mapping $H: L^{2}(\Omega) \rightarrow L^{2}(\Omega)$, then every solution $u^{*}$ to (3.1) belongs to $D(-\Delta)$.

3. Under the additional assumption

$$
\lambda_{1}^{-1 / 2}|l|_{2} L_{a} \Upsilon<m-\lambda_{1}^{-1} L_{f}-L_{H}
$$

problem (3.1) possesses a unique solution.

Proof. (i) Let us prove the first result. Consider an orthonormal Hilbert basis $\left\{w_{j}: j \geq 1\right\}$ of $L^{2}(\Omega)$ consisting of the eigenvectors associated with eigenvalues $\left\{\lambda_{j}: j \geq 1\right\}$ of the operator $-\Delta$ with zero Dirichlet boundary condition in $\Omega$. For each $n \geq 1$, let us denote $V_{n}=\operatorname{span}\left[w_{1}, \cdots, w_{n}\right]$, with the inner product $((\cdot, \cdot))$ and norm $\|\cdot\|$.

Now, we define operators $R_{n}: V_{n} \rightarrow V_{n}$, for all $n \geq 1$, as follows:

$$
\left(\left(R_{n} u, v\right)\right)=<-a(l(u)) \Delta u, v>-(f(u), v)-<H(u), v>, \quad \forall u, v \in V_{n} .
$$

Observe that the right hand side of (3.5) is a continuous linear map from $V_{n}$ to $\mathbb{R}$, thanks to the Riesz Theorem, each $R_{n} u \in V_{n}$ is well defined. In addition, by means of (3.2), the Poincaré inequality and the Lipschitz continuity of the functions $a, f$ and $H$, we deduce,

$$
\begin{aligned}
& \left(\left(R_{n} u-R_{n} \tilde{u}, v\right)\right) \\
& =<-a(l(u)) \Delta u+a(l(\tilde{u})) \Delta \tilde{u}-f(u)+f(\tilde{u})-H(u)+H(\tilde{u}), v> \\
& =<-a(l(u)) \Delta(u-\tilde{u})+(a(l(\tilde{u}))-a(l(u)) \Delta \tilde{u}, v> \\
& \quad+(f(\tilde{u})-f(u), v)+<H(\tilde{u})-H(u), v> \\
& \leq\left(M+L_{a}|l| \lambda_{1}^{-1 / 2}\|\tilde{u}\|+L_{f} \lambda_{1}^{-1}+L_{H}\right)\|\tilde{u}-u\|\|v\|,
\end{aligned}
$$

for all $u, \tilde{u}, v \in V_{n}$. Therefore,

$$
\left\|R_{n} u-R_{n} \tilde{u}\right\| \leq\left(M+L_{a}|l| \lambda_{1}^{-1 / 2}\|\tilde{u}\|+L_{f} \lambda_{1}^{-1}+L_{H}\right)\|\tilde{u}-u\|,
$$

for all $u, \tilde{u} \in V_{n}$. Namely, $R_{n}$ is continuous.

On the other hand, again by (3.2), the Poincaré inequality and the global Lipschitz continuity of $f$, we have

$$
\begin{aligned}
\left(\left(R_{n} u, u\right)\right) & =<-a(l(u)) \Delta u, u>-(f(u), u)-<H(u)-H(0), u>-<H(0), u> \\
& \geq m\|u\|^{2}-L_{f} \lambda_{1}^{-1}\|u\|^{2}-\left|f(0)\left\|\left.\Omega\right|^{1 / 2} \lambda_{1}^{-1 / 2}\right\| u\left\|-L_{H}\right\| u\left\|^{2}-\right\| H(0)\left\|_{H^{-1}(\Omega)}\right\| u \|\right. \\
& :=\left(m-L_{f} \lambda_{1}^{-1}-L_{H}\right)\|u\|^{2}-\left(f(0)|\Omega|^{1 / 2} \lambda_{1}^{-1 / 2}+\|H(0)\|_{H^{-1}(\Omega)}\right)\|u\|,
\end{aligned}
$$


for all $u \in V_{n}$. Therefore, taking

$$
\Upsilon:=\frac{\lambda_{1}^{-1 / 2}|\Omega|^{1 / 2}|f(0)|+\|H(0)\|_{H^{-1}(\Omega)}}{m-\lambda_{1}^{-1} L_{f}-L_{H}},
$$

we obtain

$$
\left(\left(R_{n} u, u\right)\right) \geq 0, \quad \forall u \in V_{n} \text { such that }\|u\|=\Upsilon .
$$

As a consequence of a corollary of the Brouwer fixed point theorem ([22, Lemma 4.3]), we deduce that for each $n \geq 1$, there exists $u_{n} \in V_{n}$ such that $R_{n}\left(u_{n}\right)=0$, with

$$
\|u\| \leq \Upsilon
$$

Therefore,

$$
<-a\left(l\left(u^{n}\right)\right) \Delta u^{n}, v>=\left(f\left(u^{n}\right), v\right)+<H\left(u^{n}\right), v>, \quad \forall v \in V_{n} .
$$

Now, using the boundedness of $\left\{u^{n}\right\}$ in $H_{0}^{1}(\Omega)$ by $\Upsilon$ and the compact embedding $H_{0}^{1}(\Omega) \hookrightarrow$ $L^{2}(\Omega)$, we can extract a subsequence $\left\{u^{n_{k}}\right\} \subset\left\{u^{n}\right\}$, such that

$$
\begin{cases}u^{n_{k}} \rightarrow u^{*}, & \text { weakly in } H_{0}^{1}(\Omega), \\ u^{n_{k}} \rightarrow u^{*}, & \text { strongly in } L^{2}(\Omega),\end{cases}
$$

where $u^{*} \in H_{0}^{1}(\Omega)$ is a solution to (3.1). To check that, we just take limit in (3.7) and use assumptions imposed on $a, l, f$ and $H$. Moreover, $u^{*}$ fulfils (3.6).

So far, we only have proved that there exists at least a solution $u^{*}$ to problem (3.1) and verifies (3.3). But this does not imply that any solution $u^{*}$ to problem (3.1) fulfils (3.3) since the uniqueness of solution is not guaranteed. Therefore, we are going to prove that any solution $u^{*}$ to problem (3.1) fulfils (3.3).

Let $u^{*}$ be a solution to (3.1). It holds

$$
\begin{aligned}
m\left\|u^{*}\right\|^{2} \leq & \left|f\left(u^{*}\right)-f(0)\left\|u^{*}|+| f(0)\right\| \Omega\right|^{1 / 2}\left|u^{*}\right| \\
& +<H\left(u^{*}\right)-H(0), u^{*}>+\|H(0)\|_{H^{-1}(\Omega)}\left\|u^{*}\right\| .
\end{aligned}
$$

Since the functions $f$ and $H$ are globally Lipschitz, by the Poincaré inequality, we obtain

$$
m\left\|u^{*}\right\|^{2} \leq\left(L_{f} \lambda_{1}^{-1}+L_{H}\right)\left\|u^{*}\right\|^{2}+\left(\left|f(0)\left\|\left.\Omega\right|^{1 / 2} \lambda_{1}^{-1 / 2}+\right\| H(0) \|_{H^{-1}(\Omega)}\right)\left\|u^{*}\right\| .\right.
$$

Therefore, each solution $u^{*}$ of problem (3.1) satisfies (3.3).

(ii) We will check that if $H: L^{2}(\Omega) \rightarrow L^{2}(\Omega)$, then every solution $u^{*}$ to (3.1) belongs to $D(-\Delta)$. In what follows, we represent $\left(u^{n}\right)^{*}=P_{n} u^{*}:=\sum_{i=1}^{n}\left(u^{*}, w_{i}\right) w_{i}$.

Since $u^{*}$ is a solution to (3.1), taking $v=-\Delta\left(u^{n}\right)^{*}$ in Definition 3.1, we deduce

$$
a\left(l\left(u^{*}\right)\right)\left|-\Delta\left(u^{n}\right)^{*}\right|^{2}=\left(f\left(u^{*}\right),-\Delta\left(u^{n}\right)^{*}\right)+\left(H\left(u^{*}\right),-\Delta\left(u^{n}\right)^{*}\right) .
$$


Using the Cauchy-Schwartz and Young inequalities, the fact that $f$ and $H$ are globally Lipschitz and (3.3), we deduce

$$
\begin{aligned}
\left(f\left(u^{*}\right),-\Delta\left(u^{n}\right)^{*}\right) & \leq \frac{1}{m}\left|f\left(u^{*}\right)\right|^{2}+\frac{m}{4}\left|-\Delta\left(u^{n}\right)^{*}\right|^{2} \\
& \leq \frac{2}{m}\left|f\left(u^{*}\right)-f(0)\right|^{2}+\frac{m}{4}\left|-\Delta\left(u^{n}\right)^{*}\right|^{2}+\frac{2}{m} \int_{\Omega}|f(0)|^{2} d x \\
& \leq \frac{2 L_{f}^{2}}{m \lambda_{1}}|| u^{*} \|^{2}+\frac{2}{m}|f(0)|^{2}|\Omega|+\frac{m}{4}\left|-\Delta\left(u^{n}\right)^{*}\right|^{2} \\
& \leq \frac{2 \lambda_{1}^{-1} L_{f}^{2} \Upsilon^{2}+2|f(0)|^{2}|\Omega|}{m}+\frac{m}{4}\left|-\Delta\left(u^{n}\right)^{*}\right|^{2},
\end{aligned}
$$

and

$$
\begin{aligned}
\left(H\left(u^{*}\right),-\Delta\left(u^{n}\right)^{*}\right) & \leq \frac{1}{m}\left|H\left(u^{*}\right)\right|^{2}+\frac{m}{4}\left|-\Delta\left(u^{n}\right)^{*}\right|^{2} \\
& \leq \frac{2 L_{H}^{2}}{m \lambda_{1}}\left\|u^{*}\right\|^{2}+\frac{2}{m}|H(0)|^{2}|\Omega|+\frac{m}{4}\left|-\Delta\left(u^{n}\right)^{*}\right|^{2} .
\end{aligned}
$$

Thus,

$$
\left|-\Delta\left(u^{n}\right)^{*}\right|^{2} \leq \frac{2}{m^{2}}\left(2 \lambda_{1}^{-1} L_{f}^{2} \Upsilon^{2}+2 \lambda_{1}^{-1} L_{H}^{2} \Upsilon^{2}+2\left(|f(0)|^{2}+|H(0)|^{2}\right)|\Omega|\right) .
$$

Then, as the sequence $\left\{P_{n} u^{*}\right\}$ is bounded in $D(-\Delta)$ and $P_{n} u^{*}$ converges to $u^{*}$ strongly in $L^{2}(\Omega)$, it holds that $u^{*} \in D(-\Delta)$.

(iii) Finally, we state the uniqueness to this problem. Assume $u_{1}$ and $u_{2}$ are two solutions of (3.1). Then

$$
\begin{aligned}
& <-a\left(l\left(u_{1}\right)\right) \Delta u_{1}+a\left(l\left(u_{2}\right)\right) \Delta u_{2}, v> \\
= & \left(f\left(u_{1}\right)-f\left(u_{2}\right), v\right)+<H\left(u_{1}\right)-H\left(u_{2}\right), v>, \quad \forall v \in H_{0}^{1}(\Omega) .
\end{aligned}
$$

Adding $\pm a\left(l\left(u_{1}\right)\right) \Delta u_{2}$ into the above equality, taking $v=u_{1}-u_{2}$, we obtain

$$
m\left\|u_{1}-u_{2}\right\|^{2} \leq\left(\lambda_{1}^{-1 / 2}|l| L_{a}\left\|u_{2}\right\|+\lambda_{1}^{-1} L_{f}+L_{H}\right)\left\|u_{1}-u_{2}\right\|^{2} .
$$

We now argue by contradiction, assume that $u_{1} \neq u_{2}$. Then, we can simplify the above expression dropping the factor $\left\|u_{1}-u_{2}\right\|^{2}$. However, using a priori estimate (3.3) for $u_{2}$, we would arrive at the opposite inequality to that one in (3.4), what is a contradiction. Therefore, $u_{1}=u_{2}$ holds. The proof of this theorem is complete.

In the sequel, our goal is to establish sufficient conditions ensuring stability of stationary solutions to (2.1). We first recall the stability definitions we will use in our analysis later.

Definition 3.3 (1) A stationary solution $u^{*}$ to (2.1) is said to be stable, if for any $\varepsilon>0, \tau \in \mathbb{R}$, there exists $\delta>0$ such that if $\varphi \in L^{2}\left((-\rho, 0] ; H_{0}^{1}(\Omega)\right)$ satisfies $\left\|\varphi-u^{*}\right\|_{L^{2}\left((-\rho, 0] ; H_{0}^{1}(\Omega)\right)}<\delta$, then 
the corresponding solution $u(\cdot ; \tau, \varphi)$ to (2.1) exists for all $t \geq \tau$, and satisfies $\left|u(t ; \tau, \varphi)-u^{*}\right|<\varepsilon$, for all $t \geq \tau$.

(2) A stationary solution $u^{*}$ to (2.1) is said to be attractive, if for every $\tau \in \mathbb{R}$ there exists $\tilde{\delta}>0$ such that for $\varphi \in L^{2}\left((-\rho, 0] ; H_{0}^{1}(\Omega)\right)$ with $\left\|\varphi-u^{*}\right\|_{L^{2}\left((-\rho, 0] ; H_{0}^{1}(\Omega)\right)}<\tilde{\delta}$, the solution $u(\cdot ; \tau, \varphi)$ to (2.1) exists for all $t \geq \tau$, and satisfies $\lim _{t \rightarrow \infty}\left|u(t ; \tau, \varphi)-u^{*}\right|=0$.

(3) A stationary solution $u^{*}$ to (2.1) is said to be asymptotically stable if it is stable and attractive.

\subsection{Analysis of stability: a direct approach}

In this section, we prove the local stability of stationary solutions for a general delay term by doing energy estimations directly. Moreover, the asymptotic stability of stationary solutions to (2.1) with bounded delay will be presented later.

Theorem 3.4 Assume the hypotheses of Theorem 3.2 and $\left(H_{4}\right)$. If $u(\cdot)$ is any solution to (2.1), and $u^{*}$ is the unique stationary solution of the same problem, then

$$
\left|u(t)-u^{*}\right|^{2} \leq\left|\varphi(0)-u^{*}\right|^{2}+\frac{C_{h}}{m}\left\|\varphi-u^{*}\right\|_{L^{2}\left((-\rho, 0] ; H_{0}^{1}(\Omega)\right)}^{2}, \quad \forall t \geq \tau,
$$

provided

$$
\frac{2 L_{a}|l|\left\|u^{*}\right\|}{\sqrt{\lambda_{1}}}+\frac{2 L_{f}}{\lambda_{1}}+\frac{C_{h}}{m} \leq m
$$

Proof. From the energy equality,

$$
\begin{aligned}
\frac{1}{2} \frac{d}{d t}\left|u(t)-u^{*}\right|^{2}= & <a(l(u(t))) \Delta u(t)-a\left(l\left(u^{*}\right)\right) \Delta u^{*}+f(u) \\
& -f\left(u^{*}\right)+h\left(t, u_{t}\right)-h\left(t, u^{*}\right), u(t)-u^{*}>.
\end{aligned}
$$

Adding $\pm a(l(u)) \Delta u^{*}$ in (3.10) and using (3.2), the Poincaré and Young inequalities, the global Lipschitz continuity of the functions $a$ and $f$, we have

$$
\begin{aligned}
\frac{d}{d t}\left|u(t)-u^{*}\right|^{2} \leq & 2 a(l(u))\left(\Delta\left(u-u^{*}\right), u(t)-u^{*}\right)+2\left(a(l(u)) \Delta u^{*}-a\left(l\left(u^{*}\right)\right) \Delta u^{*}, u(t)-u^{*}\right) \\
& +2\left(f(u)-f\left(u^{*}\right), u(t)-u^{*}\right)+2<h\left(t, u_{t}\right)-h\left(t, u^{*}\right), u(t)-u^{*}> \\
\leq & 2\left(-\frac{m}{2}+\frac{L_{a}|l|\left\|u^{*}\right\|}{\sqrt{\lambda_{1}}}+\frac{L_{f}}{\lambda_{1}}\right)\left\|u(t)-u^{*}\right\|^{2} \\
& +\frac{1}{m}\left\|h\left(t, u_{t}\right)-h\left(t, u^{*}\right)\right\|_{H^{-1}(\Omega)}^{2} .
\end{aligned}
$$

Integrating the above expression between $\tau$ and $t$, by $\left(H_{4}\right)$, we obtain

$$
\begin{aligned}
\left|u(t)-u^{*}\right|^{2} \leq & \left|\varphi(0)-u^{*}\right|^{2}+\frac{C_{h}}{m} \int_{-\rho}^{0}\left\|\varphi-u^{*}\right\|^{2} d s \\
& +2\left(-\frac{m}{2}+\frac{L_{a}|l|\left\|u^{*}\right\|}{\sqrt{\lambda_{1}}}+\frac{L_{f}}{\lambda_{1}}+\frac{C_{h}}{2 m}\right) \int_{\tau}^{t}\left\|u(s)-u^{*}\right\|^{2} d s .
\end{aligned}
$$


Neglecting the last term on the right-hand side of the above inequality since it is non-positive, it follows,

$$
\left|u(t)-u^{*}\right|^{2} \leq\left|\varphi(0)-u^{*}\right|^{2}+\frac{C_{h}}{m}\left\|\varphi-u^{*}\right\|_{L^{2}\left((-\rho, 0] ; H_{0}^{1}(\Omega)\right)}^{2}, \quad \forall t \geq \tau .
$$

The proof is complete.

Remark 3.5 Notice that, the sufficient condition (3.9) ensuring stability of the stationary solution involves its norm. It is possible to obtain another sufficient condition independently of $\left\|u^{*}\right\|$. Indeed, take into account that $\left\|u^{*}\right\| \leq\|\Upsilon\|$, (see (3.3)). If we impose

$$
\frac{2 L_{a}|l|\|\Upsilon\|}{\sqrt{\lambda_{1}}}+\frac{2 L_{f}}{\lambda_{1}}+\frac{C_{h}}{m} \leq m
$$

then condition (3.9) holds automatically, which means this condition is stronger than (3.9).

Next we will prove asymptotic stability of stationary solutions when the delay is bounded, in fact, we will prove exponential asymptotic stability.

Theorem 3.6 Under the assumptions of Theorem 3.2, also, suppose there exist $\alpha_{0}>0$ and $C(\rho)$ (which may also depend on $\left.\alpha_{0}\right)$, such that for each $\alpha \in\left(0, \alpha_{0}\right]$, it holds

$$
\int_{\tau}^{t} e^{\alpha s}\left\|h\left(s, u_{s}\right)-h\left(s, v_{s}\right)\right\|_{H^{-1}(\Omega)}^{2} d s \leq C(\rho) \int_{\tau-\rho}^{t} e^{\alpha s}\|u(s)-v(s)\|^{2} d s,
$$

and

$$
m>\frac{2 L_{a}|l|\left\|u^{*}\right\|}{\sqrt{\lambda_{1}}}+\frac{2 L_{f}}{\lambda_{1}}+\frac{C(\rho)}{m} .
$$

If problem (2.1) is driven by bounded delay, then for sufficiently small $\alpha$, we have

$$
\left|u(t)-u^{*}\right|^{2} \leq e^{-\alpha(t-\tau)}\left(\left|\varphi(0)-u^{*}\right|^{2}+\frac{C(\rho)}{m}\left\|\varphi-u^{*}\right\|_{L^{2}\left((-\rho, 0] ; H_{0}^{1}(\Omega)\right)}^{2}\right),
$$

where $u(\cdot)$ is the solution to (2.1) corresponding to the initial value $\varphi$, and $u^{*}$ is the unique stationary solution of (2.1).

Proof. Let $0<\alpha \leq \alpha_{0}$, then

$$
\frac{d}{d t} e^{\alpha t}\left|u(t)-u^{*}\right|^{2}=\alpha e^{\alpha t}\left|u(t)-u^{*}\right|^{2}+e^{\alpha t} \frac{d}{d t}\left|u(t)-u^{*}\right|^{2} .
$$

Integrating the above equality between $\tau$ and $t$, together with (3.10),

$$
\begin{aligned}
e^{\alpha t}\left|u(t)-u^{*}\right|^{2} \leq & \left|\varphi(0)-u^{*}\right|^{2} e^{\alpha \tau}+\frac{C(\rho)}{m} \int_{-\rho}^{0} e^{\alpha(s+\tau)}\left\|\varphi(s)-u^{*}\right\|^{2} d s+\frac{\alpha}{\lambda_{1}} \int_{\tau}^{t} e^{\alpha s}\left\|u(s)-u^{*}\right\|^{2} d s \\
& +\int_{\tau}^{t} e^{\alpha s}\left(-m+\frac{2 L_{a}|l|\left\|u^{*}\right\|}{\sqrt{\lambda_{1}}}+\frac{2 L_{f}}{\lambda_{1}}+\frac{C(\rho)}{m}\right)\left\|u(s)-u^{*}\right\|^{2} d s .
\end{aligned}
$$


Notice, if $\alpha$ is small enough, combined with assumption (3.12), the sum of last two terms of the above inequality keeps being negative. Therefore,

$$
\left|u(t)-u^{*}\right|^{2} \leq e^{-\alpha(t-\tau)}\left(\left|\varphi(0)-u^{*}\right|^{2}+\frac{C(\rho)}{m}\left\|\varphi-u^{*}\right\|_{L^{2}\left((-\rho, 0] ; H_{0}^{1}(\Omega)\right)}^{2}\right) .
$$

This finishes the proof.

Remark 3.7 The previous result (Theorem 3.6) is valid only for bounded delay, because condition (3.11) is not reasonable for unbounded delay, for more explanations, see [6].

\subsection{Asymptotic stability via the construction of Lyapunov functionals}

In this subsection, we will exploit the theory of Lyapunov functionals to complete our stability analysis in the case of unbounded variable delay. In general, using Lyapunov functionals (instead of Lyapunov functions) provides better stability results (cf. [19]), but the type of the delay terms must be more specific. In our case, we will restrict to the case of variable delay (cf. (3.15)), for simplicity, we will assume zero is the stationary solution. To this end, let us introduce an abstract problem, consider operators $\tilde{A}(t, \cdot): H_{0}^{1}(\Omega) \rightarrow H^{-1}(\Omega)$ and $\tilde{f}(t, \cdot): \mathbf{C}_{\infty}\left(H_{0}^{1}(\Omega)\right) \rightarrow H_{0}^{1}(\Omega)$ with $\tilde{A}(t, 0)=0$ and $\tilde{f}(t, 0)=0$. Assume

$$
\begin{cases}\frac{d u}{d t}=\tilde{A}(t, u)+\tilde{f}\left(t, u_{t}\right), & t \in[\tau, \infty), \\ u_{\tau}(\theta)=\varphi(\theta), & \theta \in(-\infty, 0],\end{cases}
$$

is a well-posed problem, its solution, belonging to $\mathbf{C}_{\infty}\left(H_{0}^{1}(\Omega)\right)$, is defined globally in time.

The stability of the trivial solution of (3.14) can be analyzed by constructing Lyapunov functionals. Moreover, if we improve the decay of the functional, we gain not only stability but asymptotic stability. Before proving the main theorem, let us recall the following well-known result (see, e.g. [23]).

Proposition 3.8 Assume that there exist a functional $V: \mathbb{R}^{+} \times \mathbf{C}_{\infty}\left(H_{0}^{1}(\Omega)\right) \rightarrow \mathbb{R}^{+}$and positive constants $\gamma_{1}, \gamma_{2}$ such that, for any $\varphi \in \mathbf{C}_{\infty}\left(H_{0}^{1}(\Omega)\right)$, the solution $u(\cdot)=u(\cdot ; \tau, \varphi)$ to (3.14) satisfies

$$
\begin{aligned}
& V\left(t, u_{t}\right) \geq \gamma_{1}|u(t)|^{2}, \quad t \geq \tau \\
& V\left(\tau, u_{\tau}\right) \leq \gamma_{2}\|\varphi\|_{\mathbf{C}_{\infty}\left(H_{0}^{1}(\Omega)\right)}^{2}
\end{aligned}
$$

Then:

(i) If $\frac{d}{d t} V\left(t, u_{t}\right) \leq 0$ for $t \geq \tau$, the trivial solution to (3.14) is stable;

(ii) If there exists a positive constant $\gamma_{3}$ such that $\frac{d}{d t} V\left(t, u_{t}\right) \leq-\gamma_{3}|u(t)|^{2}$ for $t \geq \tau$, the trivial solution to problem (3.14) is asymptotically stable. 
We will apply the above results to the following equation, which is a particular case of (3.14),

$$
\frac{d u}{d t}=a(l(u)) \Delta u+f(u)+H(u(t-\rho(t))),
$$

where $H: H_{0}^{1}(\Omega) \rightarrow H_{0}^{1}(\Omega)$ is an appropriate function. Note that, assumption (3.2) implies

$$
(a(l(u)) \Delta u, u) \leq-m\|u\|^{2}, \quad u \in H_{0}^{1}(\Omega) .
$$

Additionally, based on the condition that functions $H$ are globally Lipschitz with Lipschitz constant $L_{H}$, we have

$$
\|H(u)\| \leq L_{H}\|u\|, \quad u \in H_{0}^{1}(\Omega) .
$$

In the sequel, we will present the main result of this subsection.

Theorem 3.9 Assume that operators in (3.15) satisfy:

$$
\begin{array}{lc}
|f(u)| \leq \alpha_{1}|u|, & u \in H_{0}^{1}(\Omega), \\
\rho \in C^{1}\left(\mathbb{R} ; \mathbb{R}^{+}\right), & \rho^{\prime}(t) \leq \rho^{*}<1 .
\end{array}
$$

Then the trivial solution to (3.15) is stable (resp. asymptotically stable) provided that

$$
m \geq \frac{\alpha_{1}}{\lambda_{1}}+\frac{L_{H}}{\sqrt{2\left(1-\rho^{*}\right)} \lambda_{1}}\left(\text { resp. } m>\frac{\alpha_{1}}{\lambda_{1}}+\frac{L_{H}}{\sqrt{2\left(1-\rho^{*}\right)} \lambda_{1}}\right) .
$$

Proof. We construct the Lyapunov functional $V: \mathbb{R}^{+} \times \mathbf{C}_{\infty}\left(H_{0}^{1}(\Omega)\right)$ to (3.15) in the form

$$
V(t, \phi)=|\phi(0)|^{2}+\frac{C}{1-\rho^{*}} \int_{-\rho(t)}^{0}|\phi(\theta)|^{2} d \theta,
$$

where $C>0$ is a constant to be determined later on. Denoting by $V(t)=V\left(t, u_{t}(\cdot ; \tau, \varphi)\right)$, where $u_{t}(\cdot ; \tau, \varphi)$ is the solution to problem $(3.15)$ with initial value $\varphi$, we have

$$
V(t)=|u(t)|^{2}+\frac{C}{1-\rho^{*}} \int_{-\rho(t)}^{0}|u(t+\theta)|^{2} d \theta=|u(t)|^{2}+\frac{C}{1-\rho^{*}} \int_{t-\rho(t)}^{t}|u(s)|^{2} d s .
$$

Consequently,

$$
\begin{aligned}
\frac{d}{d t} V(t)= & 2<a(l(u)) \Delta u, u>+2(f(u)+H(u(t-\rho(t))), u(t)) \\
& +\frac{C}{1-\rho^{*}}|u(t)|^{2}-\frac{C\left(1-\rho^{\prime}(t)\right)}{1-\rho^{*}}|u(t-\rho(t))|^{2} \\
\leq & -2 m\|u(t)\|^{2}+\frac{2 \alpha_{1}}{\lambda_{1}}\|u(t)\|^{2}+\frac{2 L_{H}}{\sqrt{\lambda_{1}}}|u(t-\rho(t))|\|u(t)\| \\
& +\frac{C}{\left(1-\rho^{*}\right) \lambda_{1}}\|u(t)\|^{2}-C|u(t-\rho(t))|^{2} \\
\leq & 2\left(-m+\frac{\alpha_{1}}{\lambda_{1}}+\frac{C}{2\left(1-\rho^{*}\right) \lambda_{1}}\right)\|u(t)\|^{2}+\frac{L_{H}^{2}}{\lambda_{1} \varepsilon}|u(t-\rho(t))|^{2} \\
& +\varepsilon\|u(t)\|^{2}-C|u(t-\rho(t))|^{2},
\end{aligned}
$$


where the Poincaré and Young inequalities have been used. Pick up $\varepsilon=L_{H}^{2} / \lambda_{1} C$ so that the term containing $|u(t-\rho(t))|^{2}$ vanishes in the above expression. Then we conclude that

$$
\frac{d}{d t} V(t) \leq 2\left(-m+\frac{\alpha_{1}}{\lambda_{1}}+\frac{C}{2\left(1-\rho^{*}\right) \lambda_{1}}+\frac{L_{H}^{2}}{2 \lambda_{1} C}\right)\|u(t)\|^{2}
$$

and minimizing the coefficient in brackets in the right-hand side, which is attained for $C=$ $L_{H} \sqrt{1-\rho^{*}}$, we deduce

$$
\frac{d}{d t} V(t) \leq 2\left(-m+\frac{\alpha_{1}}{\lambda_{1}}+\frac{L_{H}}{2 \sqrt{\left(1-\rho^{*}\right)} \lambda_{1}}\right)\|u(t)\|^{2} .
$$

Then, the coefficient in the brackets in the right-hand side above is less than or equal or strictly less than zero depending on the conditions in (3.18). This, jointly with the Poincaré inequality,

implies the good control of $\frac{d}{d t} V(t)$ in order to apply Proposition 3.8. Therefore, both stability and asymptotic stability statements follow respectively.

\section{Existence of pullback attractors}

The purpose of this section is to prove some general results on the global asymptotic behavior of problem (2.1) completing those shown in the previous sections.

In order to proceed, we start with some standard notations related to dynamical systems.

Definition 4.1 Given a metric space $(X, d)$, a process $U$ on $X$ is a biparametric family of mappings $U(t, \tau): X \rightarrow X$ for $-\infty<\tau \leq t<+\infty$, with the following properties:

(i) $U(t, \tau) \in C(X ; X)$ for all $t \geq \tau$;

(ii) $U(\tau, \tau)=I d$ (the identity map) for all $\tau \in \mathbb{R}$;

(iii) $U(t, \tau)=U(t, r) U(r, \tau)$ for all $-\infty<\tau \leq r \leq t<+\infty$.

Next, we recall some useful concepts in order to study the asymptotic behavior of a process.

Definition 4.2 For a process $U$ defined on a metric space $(X, d)$, a family $\hat{B}_{0}=\left\{B_{0}(t): t \in \mathbb{R}\right\}$ of subsets of $X$ is said to be pullback absorbing for bounded sets, if, for any bounded set $B$ of $X$, and any $t$, there exists a time $T(B, t)$ such that,

$$
U(t, \tau) B \subset B_{0}(t), \quad \forall \tau \leq T(B, t) .
$$

The process $U$ is said to be $\hat{B}_{0}$-pullback asymptotically compact if, for any $t$, and any sequences $\left\{\tau_{n}\right\},\left\{x_{n}\right\} \subset X$ with $\tau_{n} \leq t, \lim _{n \rightarrow+\infty} \tau_{n}=-\infty$, and $x_{n} \in B_{0}\left(\tau_{n}\right)$, the sequence $\left\{U\left(t, \tau_{n}\right) x_{n}\right\}$ is relatively compact in $X$.

Definition 4.3 A family $\mathcal{A}=\{\mathcal{A}(t): t \in \mathbb{R}\}$ is said to be a pullback attractor for a process $U$ if: (i) Each $\mathcal{A}(t)$ is a compact subset of $X$ for all $t \in \mathbb{R}$; 
(ii) It is invariant, i.e.

$$
U(t, \tau) \mathcal{A}(\tau)=\mathcal{A}(t), \quad \forall \tau \leq t
$$

(iii) It attracts bounded sets in pullback sense, i.e., given a bounded subset $B$ of $X$,

$$
\lim _{\tau \rightarrow-\infty} \operatorname{dist}(U(t, \tau) B, \mathcal{A}(t))=0, \quad \forall t \in \mathbb{R},
$$

where $\operatorname{dist}\left(C_{1}, C_{2}\right)$ denotes the Hausdorff semi-distance between two sets $C_{1}$ and $C_{2}$, i.e.,

$$
\operatorname{dist}\left(C_{1}, C_{2}\right)=\sup _{x \in C_{1}} \inf _{y \in C_{2}} d(x, y)
$$

The proof of the following result, which is a slight variant of Theorem 1.1 in [15], can be found in $[24]$.

Theorem 4.4 Consider a family $\hat{B}_{0}=\left\{B_{0}(t): t \in \mathbb{R}\right\}$ of nonempty subsets of $X$ and a process $U$ on $X$ that is $\hat{B}_{0}$-asymptotically compact, and assume also that $\hat{B}_{0}$ is pullback absorbing for $U$. Then, the family of sets $\hat{\mathcal{A}}=\{\mathcal{A}(t): t \in \mathbb{R}\}$ given by

$$
\mathcal{A}(t)=\overbrace{B \text { bounded }} \Lambda(B, t),
$$

where

$$
\Lambda(B, t):=\bigcap_{s \leq t}{\overline{\bigcup_{\tau \leq s} U(t, \tau) B}}^{X}, \quad \forall t \in \mathbb{R},
$$

is a pullback attractor for $U$.

Remark 4.5 Observe that the pullback attractor defined by (4.1) is the minimal family of closed sets that attracts all bounded sets; i.e., if $\tilde{\mathcal{A}}=\{\tilde{\mathcal{A}}(t)\}$ also attracts bounded sets in a pullback sense and $\tilde{\mathcal{A}}(t)$ is closed for all $t$, then $\mathcal{A}(t) \subset \tilde{\mathcal{A}}(t)$.

\subsection{Pullback attractors in $L^{2}$-norm in the bounded delay case}

Notice that we have proved the well-posedness of (2.1) for every $T>\tau$, which implies, the weak solution of (2.1) is defined globally in time. Now, under the initial setting of Section 2, fulfilled with some more general assumptions, we are going to analyse the long-time behavior of solutions to problem $(2.1)$ in $L^{2}(\Omega)$ by means of the theory of pullback attractors. Throughout this section, we denote $\mathbb{R}_{d}^{2}=\left\{(t, \tau) \in \mathbb{R}^{2}: \tau \leq t\right\}$.

First of all, thanks to Theorem 2.7, the mapping $U: \mathbb{R}_{d}^{2} \times \mathbf{C}_{\rho}\left(L^{2}(\Omega)\right) \rightarrow \mathbf{C}_{\rho}\left(L^{2}(\Omega)\right)$, defined by

$$
U(t, \tau) \varphi=u_{t}(\cdot ; \tau, \varphi) \quad \forall \varphi \in \mathbf{C}_{\rho}\left(L^{2}(\Omega)\right), \quad \forall \tau \leq t,
$$

is obviously a process on $\mathbf{C}_{\rho}\left(L^{2}(\Omega)\right)$, where $0<\rho<\infty$ and $u_{t}(\cdot ; \tau, \varphi)$, as usual, is the weak solution to $(2.1)$. 
From now on, besides (2.3)-(2.4), we assume that function $f$ also satisfies

$$
f(s) s \leq \alpha|s|^{2}+\beta, \quad \forall s \in \mathbb{R},
$$

where $\alpha \in\left[0, m \lambda_{1} / 2\right)$ and $\beta \geq 0$. Observe that if the constant $C_{f}$ appearing in assumption (2.3) belongs to $\left[0, m \lambda_{1} / 2\right)$, this new assumption would be redundant.

Now we have the following result, which is essentially an estimation for absorbing set.

Lemma 4.6 Suppose that function $a$ is locally Lipschitz and satisfies (2.2), $f \in C(\mathbb{R})$ fulfilling (2.3), (2.4) and (4.3), $h:[\tau, T] \times \mathbf{C}_{\rho}\left(L^{2}(\Omega)\right) \rightarrow L^{2}(\Omega)$ satisfying $\left(H_{1}\right)-\left(H_{2}\right)$ with $X=Y=L^{2}(\Omega)$, $l \in L^{2}(\Omega)$ and $\varphi \in \mathbf{C}_{\rho}\left(L^{2}(\Omega)\right)$. In addition, suppose that for each $k_{0}>0$, there exists $M(\rho)>0$ (which may also depend on $\left.k_{0}\right)$, such that for all $k \in\left(0, k_{0}\right]$,

$$
\int_{\tau}^{t} e^{k s} \mid h\left(s,\left.u_{s}\right|^{2} d s \leq L_{h}^{2} M(\rho) \int_{\tau-\rho}^{t} e^{k s}|u(s)|^{2} d s, \text { for } u \in C\left([\tau-\rho, T] ; L^{2}(\Omega)\right) .\right.
$$

Then, the solution $u(\cdot)=u(\cdot ; \tau, \varphi)$ to problem (2.1) fulfils

$$
\begin{aligned}
\left\|u_{t}\right\|_{\mathbf{C}_{\rho}\left(L^{2}(\Omega)\right)}^{2} \leq & \left(\|\varphi\|_{\mathbf{C}_{\rho}\left(L^{2}(\Omega)\right)}^{2}+\frac{L_{h}^{2} M(\rho)}{\lambda m \lambda_{1}}\|\varphi\|_{\mathbf{C}_{\rho}\left(L^{2}(\Omega)\right)}^{2}\right) \\
& \times\left(e^{-\lambda(t-\tau-\rho)}+e^{-\left(\lambda-\frac{L_{h}^{2} M(\rho)}{\lambda m \lambda_{1}}\right)(t-\tau-\rho)}\right) \\
& +\frac{2 \beta|\Omega|}{\lambda}+\frac{2 \beta|\Omega| L_{h}^{2} M(\rho)}{\lambda_{1} m \lambda^{2}-\lambda L_{h}^{2} M(\rho)},
\end{aligned}
$$

where $\lambda=m \lambda_{1}-2 \alpha>0$ satisfying $\lambda>\frac{L_{h}^{2} M(\rho)}{m \lambda_{1}}$.

Proof. From the energy equality, the Cauchy-Schwartz inequality, (2.2) and (4.3), we deduce

$$
\frac{d}{d t}|u(t)|^{2}+2 m\|u(t)\|^{2} \leq 2 \alpha|u(t)|^{2}+2 \beta|\Omega|+\left|h\left(t, u_{t}\right)\right||u(t)| .
$$

Applying the Poincaré and Young inequalities, $\left(H_{2}\right)-\left(H_{3}\right)$ to the above expression, we obtain,

$$
\frac{d}{d t}|u(t)|^{2}+\left(m \lambda_{1}-2 \alpha\right)|u(t)|^{2} \leq 2 \beta|\Omega|+\frac{1}{m \lambda_{1}}\left|h\left(t, u_{t}\right)\right|^{2}
$$

By (4.4), it follows that

$$
\begin{aligned}
|u(t)|^{2} \leq & |\varphi(0)|^{2} e^{-\lambda(t-\tau)}+\frac{2 \beta|\Omega|}{\lambda}+\frac{1}{m \lambda_{1}} \int_{\tau}^{t} e^{-\lambda(t-s)}\left|h\left(s, u_{s}\right)\right|^{2} d s \\
\leq & |\varphi(0)|^{2} e^{-\lambda(t-\tau)}+\frac{2 \beta|\Omega|}{\lambda}+\frac{L_{h}^{2} M(\rho)}{\lambda m \lambda_{1}}\|\varphi\|_{\mathbf{C}_{\rho}\left(L^{2}(\Omega)\right)}^{2} e^{-\lambda(t-\tau)} \\
& +\frac{L_{h}^{2} M(\rho)}{\lambda_{1} m} \int_{\tau}^{t} e^{-\lambda(t-s)}|u(s)|^{2} d s .
\end{aligned}
$$


Gronwall's Lemma implies for all $t \geq \tau$,

$$
\begin{aligned}
|u(t)|^{2} \leq & \left(\|\varphi\|_{\mathbf{C}_{\rho}\left(L^{2}(\Omega)\right)}^{2}+\frac{L_{h}^{2} M(\rho)}{\lambda m \lambda_{1}}\|\varphi\|_{\mathbf{C}_{\rho}\left(L^{2}(\Omega)\right)}^{2}\right) \\
& \times\left(e^{-\lambda(t-\tau)}+e^{-\left(\lambda-\frac{L_{h}^{2} M(\rho)}{\lambda m \lambda_{1}}\right)(t-\tau)}\right) \\
& +\frac{2 \beta|\Omega|}{\lambda}+\frac{2 \beta|\Omega| L_{h}^{2} M(\rho)}{\lambda_{1} m \lambda^{2}-\lambda L_{h}^{2} M(\rho)} .
\end{aligned}
$$

Then,

$$
\begin{aligned}
\left\|u_{t}\right\|_{\mathbf{C}_{\rho}\left(L^{2}(\Omega)\right)}^{2} \leq & \left(\|\varphi\|_{\mathbf{C}_{\rho}\left(L^{2}(\Omega)\right)}^{2}+\frac{L_{h}^{2} M(\rho)}{\lambda m \lambda_{1}}\|\varphi\|_{\mathbf{C}_{\rho}\left(L^{2}(\Omega)\right)}^{2}\right) \\
& \times\left(e^{-\lambda(t-\tau-\rho)}+e^{-\left(\lambda-\frac{L_{h}^{2} M(\rho)}{\lambda m \lambda_{1}}\right)(t-\tau-\rho)}\right) \\
& +\frac{2 \beta|\Omega|}{\lambda}+\frac{2 \beta|\Omega| L_{h}^{2} M(\rho)}{\lambda_{1} m \lambda^{2}-\lambda L_{h}^{2} M(\rho)},
\end{aligned}
$$

for all $t \geq \tau+\rho$. We note that if $\tau \leq t<\tau+\rho$, then we obtain the following estimation

$$
\begin{aligned}
\left\|u_{t}(\theta)\right\|_{\mathbf{C}_{\rho}\left(L^{2}(\Omega)\right)}^{2} & \leq \sup _{\theta \in[-(t-\tau), 0]}|u(t+\theta)|^{2}+\sup _{\theta \in[-\rho,-(t-\tau)]}|u(t+\theta)|^{2} \\
& \leq \sup _{s \in[\tau, t]}|u(s)|^{2}+\sup _{s \in[t-\rho, \tau]}|u(s)|^{2} \\
& \leq \sup _{s \in[\tau, t]}|u(s)|^{2}+\|\varphi\|_{\mathbf{C}_{\rho}\left(L^{2}(\Omega)\right)}^{2} \\
& \leq \sup _{s \in[\tau, t]}|u(s)|^{2}+e^{-\lambda(t-\tau-\rho)}\|\varphi\|_{\mathbf{C}_{\rho}\left(L^{2}(\Omega)\right)}^{2} .
\end{aligned}
$$

Combining (4.6) and (4.7), (4.5) holds true.

Defining now

$$
S=\frac{4 \beta|\Omega|}{\lambda}+\frac{4 \beta|\Omega| L_{h}^{2} M(\rho)}{\lambda_{1} m \lambda^{2}-\lambda L_{h}^{2} M(\rho)},
$$

we are able to prove the existence of an absorbing family in the space $\mathbf{C}_{\rho}\left(L^{2}(\Omega)\right)$.

Lemma 4.7 Under the assumptions of Lemma 4.6, the family $\hat{B}_{0}=\{B(t), t \in \mathbb{R}\}$, where $B(t)=$ $B_{L^{2}(\Omega)}(0, S)$, for all $t \in \mathbb{R}$, is pullback absorbing for bounded sets in $\mathbf{C}_{\rho}\left(L^{2}(\Omega)\right)$.

Proof. To see that, we have to prove for every bounded set $D \in \mathbf{C}_{\rho}\left(L^{2}(\Omega)\right)$, and for every fixed $t \in \mathbb{R}$, there exists $T=T(t, D)$, such that

$$
\sup _{\varphi \in D}\left\|u_{t}(\cdot ; \tau, \varphi)\right\|_{\mathbf{C}_{\rho}\left(L^{2}(\Omega)\right)}^{2} \leq S, \quad \forall \tau \geq T .
$$

As $D$ is a bounded set in $\mathbf{C}_{\rho}\left(L^{2}(\Omega)\right)$, there is a constant $d>0$, such that

$$
\|\varphi\|_{\mathbf{C}_{\rho}\left(L^{2}(\Omega)\right)} \leq d, \quad \text { for all } \varphi \in D
$$


Thanks to Lemma 4.6, we have

$$
\left\|u_{t}(\cdot ; \varphi, \tau)\right\|_{\mathbf{C}_{\rho}\left(L^{2}(\Omega)\right)}^{2} \leq 2\left(d^{2}+\frac{L_{h}^{2} M(\rho) d^{2}}{\lambda m \lambda_{1}}\right) e^{-\left(\lambda-\frac{L_{h}^{2} M(\rho)}{\lambda m \lambda_{1}}\right)(t-\tau-\rho)}+\frac{S}{2},
$$

for any $t \geq \tau$.

Now we do estimation on the first term of the right hand side of the above inequality. It is clear that there exists a constant $T=T(D, t)$, such that

$$
2\left(d^{2}+\frac{L_{h}^{2} M(\rho) d^{2}}{\lambda m \lambda_{1}}\right) e^{-\left(\lambda-\frac{L_{h}^{2} M(\rho)}{\lambda m \lambda_{1}}\right)(t-\tau-\rho)} \leq \frac{S}{2}, \quad \forall \tau \leq T .
$$

Thus, we finish the proof of this lemma.

To conclude with the existence of pullback attractors, we will prove that the process $U$ given by (4.2) is pullback asymptotically compact. First, we prove the following general result.

Lemma 4.8 Let $B \subset \mathbf{C}_{\rho}\left(L^{2}(\Omega)\right)$ be bounded, $\varphi^{n} \in B$ and $\varphi^{n} \rightarrow \varphi$ weakly in $H^{-1}(\Omega), \varphi^{n}(0) \rightarrow$ $\varphi(0)$ weakly in $L^{2}(\Omega)$. Then, denoting $u^{n}(\cdot):=u_{t}\left(\cdot ; \tau, \varphi^{n}\right)$, there exist a subsequence $\left\{u^{n_{k}}(\cdot)\right\}$ and a function $u(\cdot)$ such that $\left\{u^{n_{k}}(\cdot)\right\}$ converges to $u(\cdot)$ in $C\left([r, T] ; L^{2}(\Omega)\right)$ for all $\tau<r<T$. If moreover, $\varphi^{n} \rightarrow \varphi$ in $\mathbf{C}_{\rho}\left(L^{2}(\Omega)\right)$, then $u^{n_{k}} \rightarrow u$ in $C\left([\tau-\rho, T] ; L^{2}(\Omega)\right)$, for all $T>\tau$, and $u$ is a solution of (2.1) corresponding to the initial value $\varphi$.

Proof. Following the same lines of the proof of Theorem 2.7, we have

$$
\left\{\begin{array}{l}
u^{n} \rightarrow u \text { weakly in } L^{2}\left((\tau, T] ; H_{0}^{1}(\Omega)\right), \\
\left(u^{n}\right)^{\prime} \rightarrow u^{\prime} \text { weakly in } L^{2}\left((\tau, T] ; H^{-1}(\Omega)\right), \\
u^{n} \rightarrow u \text { strongly in } L^{2}\left((\tau, T] ; L^{2}(\Omega)\right), \\
u^{n}(t) \rightarrow u(t) \text { strongly in } L^{2}(\Omega), \text { a.e. } t \in[\tau, T], \\
f\left(u^{n}(t)\right) \rightarrow f(u(t)) \text { weakly in } L^{2}\left((\tau, T] ; L^{2}(\Omega)\right), \\
a\left(l\left(u^{n}\right)\right) \Delta u^{n} \rightarrow a(l(u)) \Delta u \text { weakly in } L^{2}\left((\tau, T] ; H_{0}^{1}(\Omega)\right), \\
h\left(t, u_{t}^{n}\right) \rightarrow \xi_{h} \text { weakly in } L^{2}\left((\tau, T] ; L^{2}(\Omega)\right) .
\end{array}\right.
$$

Lemma 4.6 implies that $\left\|u_{t}^{n}\right\|_{\mathbf{C}_{\rho}\left(L^{2}(\Omega)\right)}$ is uniformly bounded on $[\tau, T]$, therefore, the sequence $\left\{u^{n}(t)\right\}$ is uniformly bounded on $[\tau, T]$. This together with the compact embedding $L^{2}(\Omega) \hookrightarrow$ $H^{-1}(\Omega)$, and the Ascoli-Arzelà theorem, leads $u^{n} \rightarrow u$ in $C\left([\tau, T] ; H^{-1}(\Omega)\right)$. Then a standard argument implies that $u^{n}\left(t_{n}\right) \rightarrow u\left(t_{0}\right)$ weakly in $L^{2}(\Omega)$ for any sequence $t_{n} \rightarrow t_{0}, t_{n}, t_{0} \in[\tau, T]$, thus

$$
\left|u\left(t_{0}\right)\right|^{2} \leq \liminf _{n \rightarrow \infty}\left|u^{n}\left(t_{n}\right)\right|^{2} .
$$

In order to prove $\left\{u^{n_{k}}\right\}$ converges to $u$ in $C\left([r, T] ; L^{2}(\Omega)\right)$, we need to check $u^{n}\left(t_{n}\right) \rightarrow u\left(t_{0}\right)$ strongly in $L^{2}(\Omega)$ for any sequence $\left\{t_{n}\right\}_{n \in \mathbb{N}}, t_{0} \in[r, T]$, for any $r \in[\tau, T]$. To this end, it is enough to ensure

$$
\limsup _{n \rightarrow \infty}\left|u^{n}\left(t_{n}\right)\right|^{2} \leq\left|u\left(t_{0}\right)\right|^{2}
$$


It is not difficult to see that the functions

$$
J_{n}(t)=\frac{1}{2}\left|u^{n}(t)\right|^{2}-2 \beta|\Omega| t-C_{1} L_{h}^{2}(t-\tau), \quad \tau \leq t \leq T,
$$

are continuous and non-increasing in $[\tau, T]$, where we have used the notation that $\left\|u_{t}\right\|_{\mathbf{C}_{\rho}\left(L^{2}(\Omega)\right)}^{2} \leq$ $C_{1}$. Passing to the limit, we obtain that $u(\cdot)$ is a solution of the following problem

$$
\left\{\begin{array}{l}
\frac{d u}{d t}-a(l(u)) \Delta u=f(u)+\xi_{h}(t), \\
u_{\tau}(0)=\varphi(0), \quad u_{0}=\varphi \text { in } H^{-1}(\Omega) .
\end{array}\right.
$$

Notice that, $h\left(t, u_{t}^{n}\right) \rightarrow \xi_{h}(t)$ weakly in $L^{2}\left((\tau, T] ; L^{2}(\Omega)\right)$ implies that

$$
\int_{\tau}^{t}\left|\xi_{h}(s)\right|^{2} d s \leq \liminf _{n \rightarrow \infty} \int_{\tau}^{t}\left|h\left(s, u_{s}^{n}\right)\right|^{2} d s \leq C_{1} L_{h}^{2}(t-\tau) .
$$

Therefore, the continuous function

$$
J(t)=\frac{1}{2}|u(t)|^{2}-2 \beta|\Omega| t-C_{1} L_{h}^{2}(t-\tau)
$$

is also non-increasing in $[\tau, T]$. Moreover, (4.8) implies, passing to a subsequence, that $J_{n}(t) \rightarrow$ $J(t)$ for a.a. $t \in(\tau, T]$. Therefore, by a technical result ([9], Lemma 11), we have (4.10). Applying now a diagonal argument, we prove that the result is valid in an arbitrary interval $\tau \leq r \leq T$.

Assume now that in addition, $\varphi^{n} \rightarrow \varphi$ in $\mathbf{C}_{\rho}\left(L^{2}(\Omega)\right)$. Then, arguing as before, one check that $u^{n} \rightarrow u$ in $C\left([\tau-\rho, T] ; L^{2}(\Omega)\right)$. Hence, it follows that $h\left(t, u_{t}^{n}\right) \rightarrow \xi_{h}$, and then $u$ is a solution of (2.1) corresponding to the initial value $\varphi$.

Lemma 4.9 Assume conditions of Lemma 4.8 hold. Then the process $U$ is $\hat{B}_{0}$-pullback asymptotically compact, where $\hat{B}_{0}$ is defined in Lemma 4.7 .

Proof. Let $\varphi_{n} \in B_{L^{2}(\Omega)}(0, S)$ and $\tau_{n} \leq t$, then, we have to prove $U\left(t, \tau_{n}\right) \varphi_{n}$ is relatively compact in $\mathbf{C}_{\rho}\left(L^{2}(\Omega)\right)$ as $\tau_{n} \rightarrow-\infty$. Denote, for short, $u_{t}^{n}=U\left(t, \tau_{n}\right) \varphi_{n}$ and $B=B_{L^{2}(\Omega)}(0, S)$. For each fixed $t$, we first choose $T:=\tilde{t}(t, B)$, such that $U(t, \tau) B \subset B$ for all $\tau \leq \min \{t,-T\}$. Then, for this fixed $T$, there exists $\tilde{t}(t-T, B)$ such that, for all $\tau \leq-\tilde{t}(t-T, B)$, we have

$$
U(t-T, \tau) B \subset B .
$$

Thus, since $\tau_{n} \rightarrow-\infty$, there exists $n_{0} \in \mathbb{N}$ such that, for all $n \geq n_{0}$, we have $\tau_{n} \leq \min \{-\tilde{t}(t-$ $T, B), t-T\}$,

$$
U\left(t, \tau_{n}\right) B=U(t, t-T) U\left(t-T, \tau_{n}\right) B \subset U(t, t-T) B .
$$

Then $u_{t}^{n}=U(t, t-T) \xi_{n}^{T}$, where $\xi_{n}^{T} \in B$. Let $u^{n}(\cdot)$ be a sequence of solutions such that $u_{t-T}^{n}=\xi_{n}^{T}$, namely, $u^{n}(\cdot+t-T)=\xi_{n}^{T}(\cdot)$ in $L^{2}(\Omega)$.

In a similar way as in the proof of Lemma 4.8 , it follows that $\left\{u^{n}\right\}$ converges to some functions $u$ in the sense of (4.8). Then, Lemma 4.8 implies, moreover, that

$$
u^{n} \rightarrow u \quad \text { in } \quad C\left([r, t], L^{2}(\Omega)\right), \quad \text { for all } t-T<r<t .
$$

Hence, if we take $T>\rho$, then we obtain $\left\{u_{t}^{n}\right\}$ converges to $u_{t}$ in $\mathbf{C}_{\rho}\left(L^{2}(\Omega)\right)$. 
Theorem 4.10 Assume the conditions of Lemma 4.6, and let $B \subset \mathbf{C}_{\rho}\left(L^{2}(\Omega)\right)$ be a bounded set, $\varphi^{n} \in B$ and $\varphi^{n} \rightarrow \varphi$ weakly in $H^{-1}(\Omega), \varphi^{n}(0) \rightarrow \varphi(0)$ weakly in $L^{2}(\Omega)$. Then the process $U$ defined in $\mathbf{C}_{\rho}\left(L^{2}(\Omega)\right)$ associated to (2.1) has a pullback attractor $\hat{\mathcal{A}}=\{\mathcal{A}(t)\}$.

Proof. The existence of pullback attractor is a direct consequence of Theorem 4.4, Lemmas 4.6 and 4.9 .

In general, we cannot obtain much more information about the structure of pullback attractors but just proved to exist. In fact, such attractor may have a complex structure, however, in some cases we can provide more details of the geometrical structure of this set. Indeed, in the case described below, we will be able to prove that it becomes a singleton $u^{*}$, which means the solutions are attracted by a single point, the unique stationary solution $u^{*}$.

The result is based on Theorem 3.6.

Lemma 4.11 Assume the conditions of Theorem 3.2 and $\varphi \in C\left([-\rho, 0] ; L^{2}(\Omega)\right)$, also, suppose that for each $\kappa_{0}>0$, there exists $K(\rho)>0$ (which may also depend on $\kappa_{0}$ ), such that for each $\kappa \in\left(0, \kappa_{0}\right]$, it holds

$$
\int_{\tau}^{t} e^{\kappa s}\left|h\left(s, u_{s}\right)-h\left(s, v_{s}\right)\right|^{2} d s \leq K(\rho) \int_{\tau-\rho}^{t} e^{\kappa s}|u(s)-v(s)|^{2} d s, \text { for } u, v \in C\left([\tau-\rho, t] ; L^{2}(\Omega)\right), \tau \leq t,
$$

and

$$
m \lambda_{1}>\frac{L_{a}^{2}|l|^{2}\left\|u^{*}\right\|^{2}+2 m L_{f}+m+K(\rho) m}{m} .
$$

If problem (2.1) is driven by bounded delay, then for sufficiently small $\kappa$, we have

$$
\left|u(t)-u^{*}\right|^{2} \leq e^{-\kappa(t-\tau)}\left(\left|\varphi(0)-u^{*}\right|^{2}+K(\rho)\left\|\varphi-u^{*}\right\|_{L^{2}\left((-\rho, 0] ; L^{2}(\Omega)\right)}^{2}\right),
$$

where $u(\cdot)$ is the solution to problem (2.1) corresponding to the initial value $\varphi$, and $u^{*}$ is the unique stationary solution of (2.1).

Proof. This proof follows the same lines of Theorem 3.6. Let $0<\kappa \leq \kappa_{0}$, then

$$
\frac{d}{d t} e^{\kappa t}\left|u(t)-u^{*}\right|^{2}=\kappa e^{\kappa t}\left|u(t)-u^{*}\right|^{2}+e^{\kappa t} \frac{d}{d t}\left|u(t)-u^{*}\right|^{2}
$$

Using energy estimation, by (3.2) and the Young inequality, we have

$$
\begin{aligned}
\frac{d}{d t}\left|u(t)-u^{*}\right| \leq & -2 m\left\|u(t)-u^{*}\right\|^{2}+2 L_{a}|l|\left|u(t)-u^{*}\right|\left\|u^{*}\right\|\left\|u(t)-u^{*}\right\| \\
& +2 L_{f}\left|u(t)-u^{*}\right|^{2}+\left|h\left(t, u_{t}\right)-h\left(t, u^{*}\right)\right|^{2}+\left|u(t)-u^{*}\right|^{2} \\
\leq & \left(-m \lambda_{1}+\frac{1}{m} L_{a}^{2}|l|^{2}\left\|u^{*}\right\|^{2}+2 L_{f}+1\right)\left|u(t)-u^{*}\right|^{2}+\left|h\left(t, u_{t}\right)-h\left(t, u^{*}\right)\right|^{2} .
\end{aligned}
$$


Integrating (4.13) between $\tau$ and $t$, together with the above expression and (4.11)-(4.12), we have

$$
\begin{aligned}
e^{\kappa t}\left|u(t)-u^{*}\right|^{2} \leq & \left|\varphi(0)-u^{*}\right|^{2} e^{\kappa \tau}+K(\rho) e^{\kappa \tau}\left\|\varphi-u^{*}\right\|_{L^{2}\left((-\rho, 0] ; L^{2}(\Omega)\right)}^{2}+\kappa \int_{\tau}^{t} e^{\kappa s}\left|u(s)-u^{*}\right|^{2} d s \\
& +\int_{\tau}^{t} e^{\kappa s}\left(-m \lambda_{1}+\frac{1}{m} L_{a}^{2}|l|^{2}\left\|u^{*}\right\|^{2}+2 L_{f}+1+K(\rho)\right)\left|u(s)-u^{*}\right|^{2} d s
\end{aligned}
$$

Notice, if $\kappa$ is small enough, combine with assumption (4.12), the sum of last two terms of the above inequality keeps being negative. Therefore,

$$
\left|u(t)-u^{*}\right|^{2} \leq e^{-\kappa(t-\tau)}\left(\left|\varphi(0)-u^{*}\right|^{2}+K(\rho)\left\|\varphi-u^{*}\right\|_{L^{2}\left((-\rho, 0] ; L^{2}(\Omega)\right)}^{2}\right) .
$$

This finishes the proof.

Theorem 4.12 Assume the same conditions of Lemma 4.11. Then the process $U$ possesses a pullback attractor $\mathcal{A}=\left\{u^{*}\right\}$, which is a singleton. Moreover, it is also a forward attractor.

Proof. From Lemma 4.11, it follows there exists a unique stationary solution $u^{*}$, such that for all $\tau \in \mathbb{R}, \forall \varphi \in L^{2}\left((-\rho, 0] ; L^{2}(\Omega)\right)$, the corresponding solution, $u(\cdot)=u(\cdot ; \tau, \varphi)$ of problem $(2.1)$ satisfies

$$
\left|u(t)-u^{*}\right|^{2} \leq e^{-\kappa(t-\tau)}\left(\left|\varphi(0)-u^{*}\right|^{2}+K(\rho)\left\|\varphi-u^{*}\right\|_{L^{2}\left((-\rho, 0] ; L^{2}(\Omega)\right)}^{2}\right),
$$

which implies, for each fixed $t$, and any bounded set $\varphi \in B$,

$$
\left.\operatorname{dist}(U(t, \tau)) B, u^{*}\right) \rightarrow 0 \quad \text { as } \quad \tau \rightarrow-\infty
$$

Therefore, $\left\{u^{*}\right\}$ attracts every bounded set of $C\left([-\rho, 0] ; L^{2}(\Omega)\right)$, thanks to the property of minimal attractor (Remark 4.5), $\mathcal{A}(t) \subset\left\{u^{*}\right\}$, immediately, $\mathcal{A}(t)=\left\{u^{*}\right\}$. Further, estimation (4.14) also guarantees that $\left\{u^{*}\right\}$ is forward attracting for every bounded set in $C\left([-\rho, 0] ; L^{2}(\Omega)\right)$, and then it is a forward attractor.

\subsection{Pullback attractors in $L^{2}$-norm in the unbounded delay case}

There are several phase spaces which allow us to deal with unbounded delay. For example, in the previous 3 sections, we have proved all of results in the phase space $\mathbf{C}_{\infty}\left(L^{2}(\Omega)\right)$. However, using this phase space, we are not able to prove asymptotic compactness of process $U$. To overcome this difficulty, here, we will consider the space

$$
E_{\gamma}=\left\{\varphi \in C\left((-\infty, 0] ; L^{2}(\Omega)\right): \exists \lim _{s \rightarrow-\infty} e^{\gamma s} \varphi(s) \in L^{2}(\Omega)\right\}, \quad \forall \gamma>0,
$$

which is a Banach space endowed with the norm

$$
\|\varphi\|_{\gamma}:=\sup _{s \in(-\infty, 0]} e^{\gamma s}|\varphi(s)|
$$


Therefore, our process $U: \mathbb{R}_{d}^{2} \times E_{\gamma} \rightarrow E_{\gamma}$ is defined by

$$
U(t, \tau) \varphi=u_{t}(\cdot ; \tau, \varphi) \quad \forall \varphi \in E_{\gamma}, \quad \forall \tau \leq t
$$

With the help of the exponential appearing in this phase space, asymptotic compactness of the process $U$ can be presented. Also, the global existence of weak solution in time of problem (2.1) with unbounded delay in $E_{\gamma}$ can be proved under appropriate conditions.

Proposition 4.13 Assume that function a is locally Lipschitz and (2.2) holds, $f \in C(\mathbb{R})$ satisfying (2.4) and (4.3), $l \in L^{2}(\Omega)$. Also suppose $h: \mathbb{R} \times E_{\gamma} \rightarrow L^{2}(\Omega)$, fulfilling $\left(H_{2}\right)-\left(H_{3}\right)$ with $X=Y=L^{2}(\Omega)$ (but slightly modifying condition $\left(H_{3}\right)$, i.e., replace $\mathbf{C}_{\rho}(X)$ with $E_{\gamma}(X)$ ).

Let

$$
\lambda:=\lambda_{1} m-4 \alpha>0, \quad 2 \gamma>\lambda>\frac{L_{h}^{2}}{m \lambda_{1}} .
$$

Then, the following estimations hold for any solution $u(\cdot)=u(\cdot ; \tau, \varphi)$ to problem (2.1) for all $t \geq \tau$,

$$
\left\|u_{t}\right\|_{\gamma}^{2} \leq 2 e^{-\left(\lambda-\frac{L_{h}^{2}}{m \lambda_{1}}\right)(t-\tau)}\|\varphi\|_{\gamma}^{2}+\frac{2 \beta|\Omega|}{\lambda}+\frac{2 L_{h}^{2} \beta|\Omega|}{\lambda\left(\lambda m \lambda_{1}-L_{h}^{2}\right)}
$$

and

$$
\begin{aligned}
\frac{m}{2} \int_{\tau}^{t}\|u(s)\|^{2} d s \leq & e^{-\lambda(t-\tau)}|u(\tau)|^{2}+2 e^{-\left(\lambda-\frac{L_{h}^{2}}{m \lambda_{1}}\right)(t-\tau)}\|\varphi\|_{\gamma}^{2} \\
& +\frac{2 \beta|\Omega|}{\lambda} e^{\lambda(t-\tau)}+\frac{L_{h}^{2}}{\lambda \lambda_{1} m}\left(\frac{2 \beta|\Omega|}{\lambda}+\frac{2 L_{h}^{2} \beta|\Omega|}{\lambda\left(\lambda_{1} m \lambda-L_{h}^{2}\right)}\right) e^{\lambda(t-\tau)}
\end{aligned}
$$

Proof. Multiply $(2.1)$ by $u(t)$, by $(2.2),(4.3),\left(H_{2}\right)-\left(H_{3}\right)$, the Young and Poincaré inequalities, we have

$$
\frac{d}{d t}|u(t)|^{2}+\left(\frac{\lambda_{1} m}{2}-2 \alpha\right)|u(t)|^{2}+\frac{m}{2}\|u(t)\|^{2} \leq 2 \beta|\Omega|+\frac{L_{h}^{2}}{m \lambda_{1}}\left\|u_{t}\right\|_{\gamma}^{2},
$$

integrating between $\tau$ and $t$, by (4.16), it follows

$$
\begin{aligned}
|u(t)|^{2}+\frac{m}{2} \int_{\tau}^{t} e^{-\lambda(t-s)}\|u(s)\|^{2} d s \leq & |u(\tau)|^{2} e^{-\lambda(t-\tau)}+\frac{2 \beta|\Omega|}{\lambda} e^{-\lambda(t-\tau)} \\
& +\frac{L_{h}^{2}}{m \lambda_{1}} \int_{\tau}^{t} e^{-\lambda(t-s)}\left\|u_{s}\right\|_{\gamma}^{2} d s .
\end{aligned}
$$

Further, neglecting the second term on the left hand side of (4.19),

$$
\begin{aligned}
\left\|u_{t}\right\|_{\gamma}^{2} \leq \max \left\{\sup _{\theta \in(-\infty, \tau-t]} e^{2 \gamma \theta}|\varphi(\theta+\tau-t)|^{2}, \sup _{\theta \in[\tau-t, 0]}\left(e^{2 \gamma \theta-\lambda(t+\theta-\tau)}|u(\tau)|^{2}\right.\right. \\
\left.\left.+\frac{2 \beta|\Omega|}{\lambda} e^{2 \gamma \theta-\lambda(t+\theta-\tau)}+\frac{L_{h}^{2}}{m \lambda_{1}} \int_{\tau}^{t+\theta} e^{2 \gamma \theta-\lambda(t+\theta-s)}\left\|u_{s}\right\|_{\gamma}^{2} d s\right)\right\} .
\end{aligned}
$$

By assumption (4.16), on the one hand,

$$
\sup _{\theta \in(-\infty, \tau-t]} e^{2 \gamma \theta}|\varphi(t+\theta-\tau)|^{2}=\sup _{\theta \leq 0} e^{2 \gamma(\theta-(t-\tau))}|\varphi(\theta)|^{2}=e^{-2 \gamma(t-\tau)}\|\varphi\|_{\gamma}^{2} \leq e^{-\lambda(t-\tau)}\|\varphi\|_{\gamma}^{2}
$$


On the other hand, by the same reasoning as above, we obtain

$$
\sup _{\theta \in[\tau-t, 0]} e^{2 \gamma \theta-\lambda(t+\theta-\tau)}|u(\tau)|^{2} \leq e^{-\lambda(t-\tau)}|u(\tau)|^{2}, \quad \sup _{\theta \in[\tau-t, 0]} e^{2 \gamma \theta-\lambda(t+\theta-\tau)} \frac{2 \beta|\Omega|}{\lambda} \leq \frac{2 \beta|\Omega|}{\lambda},
$$

and

$$
\sup _{\theta \in[\tau-t, 0]} \frac{L_{h}^{2}}{m \lambda_{1}} \int_{\tau}^{t+\theta} e^{2 \gamma \theta-\lambda(t+\theta-s)}\left\|u_{s}\right\|_{\gamma}^{2} d s \leq \frac{L_{h}^{2}}{m \lambda_{1}} \int_{\tau}^{t} e^{-\lambda(t-s)}\left\|u_{s}\right\|_{\gamma}^{2} d s .
$$

Collecting these estimations, we deduce that

$$
\left\|u_{t}\right\|_{\gamma}^{2} \leq e^{-\lambda(t-\tau)}\|\varphi\|_{\gamma}^{2}+\frac{2 \beta|\Omega|}{\lambda}+\frac{L_{h}^{2}}{m \lambda_{1}} \int_{\tau}^{t} e^{-\lambda(t-s)}\left\|u_{s}\right\|_{\gamma}^{2} d s .
$$

By the Gronwall lemma, we have

$$
\left\|u_{t}\right\|_{\gamma}^{2} \leq 2 e^{-\left(\lambda-\frac{L_{h}^{2}}{m \lambda_{1}}\right)(t-\tau)}\|\varphi\|_{\gamma}^{2}+\frac{2 \beta|\Omega|}{\lambda}+\frac{2 L_{h}^{2} \beta|\Omega|}{\lambda\left(\lambda m \lambda_{1}-L_{h}^{2}\right)}
$$

namely, (4.17) is true. Now we will prove (4.18), in fact, in the middle of above manipulations, we have omitted a positive term in the left-hand side of (4.19), namely $\frac{m}{2} \int_{\tau}^{t} e^{\lambda(t-s)}\left\|u_{s}\right\|^{2} d s$. Indeed, combining (4.19) and (4.20), we have

$$
\begin{aligned}
& \frac{m}{2} \int_{\tau}^{t} e^{-\lambda(t-s)}\|u(s)\|^{2} d s \\
& \quad \leq|u(\tau)|^{2} e^{-\lambda(t-\tau)}+\frac{2 \beta|\Omega|}{\lambda} e^{-\lambda(t-\tau)} \\
& \quad+\frac{L_{h}^{2}}{m \lambda_{1}} \int_{\tau}^{t}\left(\frac{2 \beta|\Omega|}{\lambda}+\frac{2 L_{h}^{2} \beta|\Omega|}{\lambda\left(\lambda m \lambda_{1}-L_{h}^{2}\right)}+2 e^{-\left(\lambda-\frac{L_{h}^{2}}{m \lambda_{1}}\right)(s-\tau)} e^{-\lambda(t-s)}\|\varphi\|_{\gamma}^{2}\right) d s
\end{aligned}
$$

thus, (4.18) is proved. The proof of this proposition is complete.

Corollary 4.14 Under the assumptions of Proposition 4.13, the family $\hat{B}_{0}=\left\{B_{0}(t), t \in \mathbb{R}\right\}$ with $B_{0}(t)=B_{E_{\gamma}}(0, \varrho)$, where

$$
\varrho^{2}=1+\frac{2 \beta|\Omega|}{\lambda}+\frac{2 L_{h}^{2} \beta|\Omega|}{\lambda\left(\lambda m \lambda_{1}-L_{h}^{2}\right)},
$$

is pullback absorbing bounded sets for the process $U$.

Proposition 4.15 Under assumptions of Corollary 4.14, the process $U$ is $\hat{B}_{0}$-asymptotically compact.

Proof. Our proof relies on the energy method. Let $t_{0} \in \mathbb{R}, u^{n}(\cdot)$ be a sequence of solutions in their respective intervals $\left[\tau_{n}, t_{0}\right]$, with initial data $\varphi^{n} \in B_{0}=B_{E_{\gamma}}(0, \varrho)$, where $\tau_{n} \rightarrow-\infty$ as $n \rightarrow+\infty$. Consider the sequence $\xi^{n}=u_{t_{0}}^{n}$. Then we will prove that this sequence is relatively compact in $E_{\gamma}$. 
Step 1: Consider two arbitrary values $0<T<\bar{T}$, we will prove that $\left.\xi^{n}\right|_{[-\bar{T}, 0]}$ is relatively compact in $C\left([-\bar{T}, 0] ; L^{2}(\Omega)\right)$. It follows from (4.17), that there exists $n_{0} \in\left(t_{0}, T\right]$ such that for all $n \geq n_{0}\left(t_{0}, T\right), \tau_{n} \leq t_{0}-T$ and

$$
\left\|u_{t}^{n}\right\|_{\gamma}^{2} \leq R, \quad \forall t \in\left[t_{0}-T, t_{0}\right], \quad \forall n \geq n_{0}\left(t_{0}, T\right)
$$

where

$$
R:=1+\frac{2 \beta|\Omega|}{\lambda}+\frac{2 L_{h}^{2} \beta|\Omega|}{\lambda\left(\lambda m \lambda_{1}-L_{h}^{2}\right)}
$$

therefore

$$
\begin{aligned}
& \left|u^{n}(t)\right|^{2} \leq R, \quad \forall t \in\left[t_{0}-T, t_{0}\right], \quad \forall n \geq n_{0}\left(t_{0}, T\right), \\
& \left\|u_{t_{0}-T}^{n}\right\|_{\gamma}^{2} \leq R, \quad \forall n \geq n_{0}\left(t_{0}, T\right) .
\end{aligned}
$$

Let $y^{n}(\cdot)=u^{n}\left(\cdot+t_{0}-T\right)$. Then, for each $n \geq 1$ such that $\tau_{n}<t_{0}-T$, the function $y^{n}(\cdot)$ is a solution on $[0, T]$ of a similar problem to $(2.1)$, namely, $h$ replaced by $\tilde{h}(s, \cdot)=h\left(s+t_{0}-T, \cdot\right)$ and with $y_{0}^{n}=u_{t_{0}-T}^{n}, y_{T}^{n}=u_{t_{0}}^{n}=\xi^{n}$. Then $\left\|y^{n}\right\|_{\gamma}$ satisfies the estimations in (4.21), for all $n \geq n_{0}\left(t_{0}, T\right)$. From (4.18) we have

$$
\left\|y^{n}\right\|_{L^{2}\left([0, T] ; H_{0}^{1}(\Omega)\right)}^{2} \leq K\left(t_{0}, T\right) .
$$

Hence, $\left\{y^{n}\right\}$ is bounded in $L^{\infty}\left((0, T] ; L^{2}(\Omega)\right)$ and $L^{2}\left((0, T] ; H_{0}^{1}(\Omega)\right)$, and $\left\{\left(y^{n}\right)^{\prime}\right\}$ is bounded in $L^{2}\left((0, T] ; H^{-1}(\Omega)\right)$. Thus, up to a subsequence (relabelled the same), there exists a function $y(\cdot)$ such that

$$
\left\{\begin{array}{l}
y^{n} \rightarrow y \text { weakly star in } L^{\infty}\left((0, T] ; L^{2}(\Omega)\right), \\
y^{n} \rightarrow y \text { weakly in } L^{2}\left((0, T] ; H_{0}^{1}(\Omega)\right), \\
\left(y^{n}\right)^{\prime} \rightarrow y^{\prime} \text { weakly in } L^{2}\left((0, T] ; H^{-1}(\Omega)\right), \\
y^{n} \rightarrow y \text { strongly in } L^{2}\left((0, T] ; L^{2}(\Omega)\right), \\
y^{n}(t) \rightarrow y(t) \text { strongly in } L^{2}(\Omega), \text { a.e. } t \in[0, T] .
\end{array}\right.
$$

Moreover, reasoning as in the proof of Lemma 4.8, we claim for any sequence $\left\{t_{n}\right\}, t_{0} \in[0, T]$ with $t_{n} \rightarrow t_{0}$,

$$
y^{n}\left(t_{n}\right) \rightarrow y\left(t_{0}\right) \quad \text { weakly in } L^{2}(\Omega) .
$$

Besides, by $\left(H_{3}\right)$ and (4.21), we obtain

$$
\int_{0}^{t}\left|\tilde{h}\left(s, y_{s}^{n}\right)\right|^{2} d s \leq L_{h}^{2} \int_{0}^{t}\left\|y_{s}^{n}\right\|_{\gamma}^{2} d s \leq C t,
$$

where $C$ does not depend on $n$ or $t$, therefore,

$$
\tilde{h}\left(t, y_{t}^{n}\right) \rightarrow v_{h}(t) \quad \text { weakly in } \quad L^{2}\left((0, T] ; L^{2}(\Omega)\right),
$$


also

$$
\begin{aligned}
& \int_{s}^{t}\left|\tilde{h}\left(r, y_{r}^{n}\right)\right|^{2} d r \leq C(t-s), \\
& \int_{s}^{t}\left|v_{h}(r)\right|^{2} d r \leq \liminf _{n \rightarrow \infty} \int_{s}^{t}\left|\tilde{h}\left(r, y_{r}^{n}\right)\right|^{2} d r \leq C(t-s), \quad \forall 0 \leq s \leq t \leq T .
\end{aligned}
$$

Then, in a standard way, one can prove $y(\cdot)$ is the unique weak solution to the problem

$$
\begin{cases}\frac{\partial y}{\partial t}-a(l(y)) \Delta y=f(y)+v_{h}(t), & \text { in } \Omega \times[0, T], \\ y(x, t)=0, & \text { on } \partial \Omega \times[0, T], \\ y(x, 0)=y_{0}(x), & \text { in } \Omega .\end{cases}
$$

Multiplying (2.1) by $u^{n}$, (4.22) by $y$, and integrating between $s$ to $t$, we obtain the energy inequality,

$$
\frac{1}{2}|z(t)|^{2}+m \int_{s}^{t}\|z(r)\|^{2} d r \leq \frac{1}{2}|z(s)|^{2}+\int_{s}^{t}(f(z(r), z(r)) d r+C(t-s), \quad 0 \leq s \leq t \leq T,
$$

where $z=y^{n}$ or $z=y$. Then, the mappings $J_{n}, J:[0, T] \rightarrow \mathbb{R}$ defined as

$$
\begin{aligned}
& J(t)=\frac{1}{2}|y(t)|^{2}+\int_{0}^{t}(f(y(r), y(r)) d r-C t, \\
& J_{n}(t)=\frac{1}{2}\left|y^{n}(t)\right|^{2}+\int_{0}^{t}\left(f\left(y^{n}(r), y^{n}(r)\right) d r-C t,\right.
\end{aligned}
$$

are non-increasing and continuous.

Analogously to the proof of Lemma 4.8, for a fixed $t_{0}>0$, using a sequence $\left\{t_{n}\right\}$ with $t_{n} \nearrow t_{0}$, we are able to establish the convergence of the norms, and therefore, jointly with the weak convergence, deduce that $y^{n} \rightarrow y$ in $C\left([\delta, T] ; L^{2}(\Omega)\right)$, for any $\delta>0$.

Now, since $T>\bar{T}$, we obtain that $\xi^{n} \rightarrow \varphi$ in $C\left([-\bar{T}, 0] ; L^{2}(\Omega)\right)$, where $\varphi(s)=y(s+T)$, for $s \in[-\bar{T}, 0]$. Repeating the same procedure for $2 \bar{T}, 3 \bar{T}, \cdots$, and using a diagonal argument, we obtain a continuous function $\varphi:(-\infty, 0] \rightarrow L^{2}(\Omega)$ and a subsequence such that $\xi^{n} \rightarrow \varphi$ in $C\left([-\bar{T}, 0] ; L^{2}(\Omega)\right)$ on every interval $[-\bar{T}, 0]$.

Moreover, since, for a fixed $T>0, u^{n}\left(s+t_{0}\right)$, with $s \in[-T, 0]$, satisfies the estimation (4.21) for any $n \geq n_{0}\left(t_{0}, T\right)$, it is clear that

$$
|\varphi(t)|^{2} \leq 1+\frac{2 \beta|\Omega|}{\lambda}+\frac{2 L_{h}^{2} \beta|\Omega|}{\lambda\left(\lambda m \lambda_{1}-L_{h}^{2}\right)} .
$$

Step 2: We claim that $\xi^{n}$ converges to $\varphi$ in $E_{\gamma}$. Indeed, we have to see that for every $\varepsilon>0$, there exists $n_{\varepsilon}$ such that

$$
\sup _{s \in(-\infty, 0]}\left|\xi^{n}(s)-\varphi(s)\right|^{2} e^{2 \gamma s} \leq \varepsilon, \quad \forall n \geq n_{\varepsilon}
$$


Fix $T_{\varepsilon}>0$ such that $e^{-2 \gamma T_{\varepsilon}} \leq \frac{\varepsilon}{4}$, and take $n_{\varepsilon} \geq n_{0}\left(t_{0}, T_{\varepsilon}\right)$ such that

$$
\left|\xi^{n}(s)-\varphi(s)\right|^{2} e^{2 \gamma s} \leq \frac{\varepsilon}{2}, \quad \forall s \in\left[-T_{\varepsilon}, 0\right] \quad \text { and } \quad \tau_{n} \leq t_{0}-T_{\varepsilon}, \quad \forall n \geq n_{\varepsilon} .
$$

(This is possible since the convergence of $\xi^{n}$ to $\varphi$ holds in compact intervals of time.) Thus, to prove (4.24), we only need to check

$$
\sup _{s \in\left(-\infty,-T_{\varepsilon}\right]}\left|\xi^{n}(s)-\varphi(s)\right|^{2} e^{2 \gamma s} \leq \frac{\varepsilon}{2}, \quad \forall n \geq n_{\varepsilon} .
$$

By (4.23) and the choice of $T_{\varepsilon}$, it is not difficult to check that, for all $k \in \mathbb{N} \cup\{0\}$, and for all $s \in\left[-\left(T_{\varepsilon}+k+1\right),-\left(T_{\varepsilon}+k\right)\right]$, it holds that

$$
e^{2 \gamma s}|\varphi(s)|^{2} \leq e^{-2 \gamma\left(T_{\varepsilon}+k\right)}|\varphi(s)|^{2} \leq \frac{\varepsilon}{4}
$$

Hence, it suffices to prove the following

$$
\sup _{s \in\left(-\infty,-T_{\varepsilon}\right]} e^{2 \gamma s}\left|\xi^{n}(s)\right|^{2} \leq \frac{\varepsilon}{4}, \quad \forall n \geq n_{\varepsilon} .
$$

Recall that $\xi^{n}$ has two parts

$$
\xi^{n}(s)= \begin{cases}\varphi^{n}\left(s+t_{0}-\tau_{n}\right), & s \in\left(-\infty, \tau_{n}-t_{0}\right], \\ u^{n}\left(s+t_{0}\right), & s \in\left[\tau_{n}-t_{0}, 0\right] .\end{cases}
$$

Thus, the proof is finished if we prove that

$$
\max \left\{\sup _{s \in\left(-\infty, \tau_{n}-t_{0}\right]} e^{2 \gamma s}\left|\varphi^{n}\left(s+t_{0}-\tau_{n}\right)\right|^{2}, \quad \sup _{s \in\left[\tau_{n}-t_{0},-T_{\varepsilon}\right]} e^{2 \gamma s}\left|u^{n}\left(s+t_{0}\right)\right|^{2}\right\} \leq \frac{\varepsilon}{4} .
$$

The first term above can be estimated as follows,

$$
\begin{aligned}
& \sup _{s \in\left(-\infty, \tau_{n}-t_{0}\right]} e^{2 \gamma s}\left|\varphi^{n}\left(s+t_{0}-\tau_{n}\right)\right|^{2} \\
& =\sup _{s \leq \tau_{n}-t_{0}} e^{2 \gamma\left(s+t_{0}-\tau_{n}\right)} e^{-2 \gamma\left(t_{0}-\tau_{n}\right)}\left|\varphi^{n}\left(s+t_{0}-\tau_{n}\right)\right|^{2} \\
& =e^{-2 \gamma\left(t_{0}-\tau_{n}\right)}\|\varphi\|_{\gamma}^{2} \\
& \leq \frac{\varepsilon}{4},
\end{aligned}
$$

thanks to the chose of $n_{\varepsilon}$. Finally, for the second term, we have

$$
\begin{aligned}
& \sup _{s \in\left[\tau_{n}-t_{0},-T_{\varepsilon}\right]} e^{2 \gamma s}\left|u^{n}\left(s+t_{0}\right)\right|^{2} \\
& =\sup _{\theta \in\left[\tau_{n}-t_{0}+T_{\varepsilon}, 0\right]} e^{2 \gamma\left(\theta-T_{\varepsilon}\right)}\left|u^{n}\left(t_{0}+\theta-T_{\varepsilon}\right)\right|^{2} \\
& \leq e^{-2 \gamma T_{\varepsilon}}\left\|u_{t_{0}-T_{\varepsilon}}^{n}\right\|_{\gamma}^{2} \\
& \leq \frac{\varepsilon}{4},
\end{aligned}
$$

where we have used (4.21) with $T=T_{\varepsilon}$. The proof is complete. 
Theorem 4.16 Assume that function a is locally Lipschitz and (2.2) holds, $f \in C(\mathbb{R})$ satisfying (2.4) and (4.3), $l \in L^{2}(\Omega)$. Also suppose $h: \mathbb{R} \times E_{\gamma} \rightarrow L^{2}(\Omega)$, fulfilling $\left(H_{2}\right)-\left(H_{3}\right)$ with $X=Y=$ $L^{2}(\Omega)$ (but slightly modifying condition $\left(H_{3}\right)$, i.e., replacing $\mathbf{C}_{\rho}(X)$ by $E_{\gamma}(X)$ ).

Let

$$
\lambda:=\lambda_{1} m-4 \alpha>0, \quad 2 \gamma>\lambda>\frac{L_{h}^{2}}{m \lambda_{1}} .
$$

Then the process $U$ defined in $E_{\gamma}$ associated to (2.1) has a pullback attractor $\hat{\mathcal{A}}=\{\mathcal{A}(t)\}$.

Remark 4.17 We end up this section with a comment on possible extension of our results. Instead of imposing condition $\left(H_{3}\right)$ on the delay term, if $h(\cdot, \cdot)$ satisfies:

$\left(H_{3}^{\prime}\right)$ There exists a function $L_{h}(\cdot) \in L_{\text {loc }}^{1}(\mathbb{R})$, such that for all $t \in(\tau, T], \alpha, \beta \in \mathbf{C}_{\rho}(X)$,

$$
\|h(t, \alpha)-h(t, \beta)\|_{Y} \leq L_{h}(t)\|\alpha-\beta\|_{\mathbf{C}_{\rho}(X)} .
$$

Then, all results in Section 4 still hold true but replacing the universe of bounded sets (to be attracted) by another appropriate universe of time-dependent families (e.g., see [25] for more details concerning this possibility).

\section{Acknowledgements}

The research of the first author has been partially supported by CSC (China Scholarship Council, No. 201906280198) for 1 year's study abroad at the University of Sevilla. The research of the second author has been partially supported by the National Natural Science Foundation of China (Nos. 11371286, 11401458) and the Natural Science Basic Research Plan in Shaanxi Province of China (No. 2019JM-165). The research of the first and third authors has been partially supported by the Spanish Ministerio de Ciencia, Innovación y Universidades (MCIU), Agencia Estatal de Investigación (AEI) and Fondo Europeo de Desarrollo Regional (FEDER) under the project PGC2018-096540-B-I00, and by Junta de Andalucía (Consejería de Economía y Conocimiento) under project US-1254251.

\section{References}

[1] M. Anguiano, P. E. Kloeden, T. Lorenz, Asymptotic behavior of nonlocal reaction-diffusion equations, Nonlinear Anal., 73 (2010), 3044-3057.

[2] M. Anguiano, T. Caraballo, J. Real, J. Valero, Pullback attractors for a nonautonomous integro-differential equation with memory in some unbounded domains, Int. J. Bifurcat. Chaos, 3 (2013), 1350042.

[3] J. W. Bebernes, C. Li, P. Talaga, Single-point blow-up for nonlocal parabolic problems, Physica D, 134 (1999), 48-60. 
[4] P. M. Berná, J. D. Rossi, Nonlocal diffusion equations with dynamical boundary conditions, Nonlinear Anal,. 195 (2020), 111751.

[5] C. Giorgi, V. Pata, A. Marzochi, Asymptotic behavior of a semilinear problem in heat conduction with memory, Nonlinear Differ. Equ. Appl., 5 (1998), 333-354.

[6] T. Caraballo, J. Real, Attractors for 2D-Navier-Stokes models with delays, J. Differ. Equations, 205 (2004), 271-297.

[7] T. Caraballo, P. Marín-Rubio, J. Valero, Autonomous and non-autonomous attractors for differential equations with delay, J. Differ. Equations, 208 (2005), 9-41.

[8] T. Caraballo, P. Marín-Rubio, J. Valero, Attractors for differential equations with unbounded delay, J. Differ. Equations, 239 (2007), 311-342.

[9] T. Caraballo, M. J. Garrido-Atienza, B. Schmalfuß, J. Valero, Global attractor for a nonautonomous integro-differential equation in materials with memory, Nonlinear Anal,. 73 (2010), 183-201.

[10] T. Caraballo, M. Herrera-Cobos, P. Marín-Rubio, Long-time behaviour of a non-autonomous parabolic equation with nonlocal diffusion and sublinear terms, Nonlinear Anal., 121 (2015), 3-18.

[11] M. Chipot, T. Savistka, Nonlocal p-Laplace equations depending on the $L^{p}$ norm of the gradient, Adv. Differential Equations, 19 (2014), 997-1020.

[12] M. Chipot, V. Valente, G. V. Caffarelli, Remarks on a nonlocal problem involving the Dirichlet energy, Rend. Sem. Mat. Univ. Padova, 110 (2003), 199-220.

[13] M. Chipot, J. F. Rodrigues, On a class of nonlocal nonlinear elliptic problems, RAIRO Modél. Math. Anal. Numér., 26 (1992), 447-467.

[14] M. Chipot, S. Zheng, Asymptotic behavior of solutions to nonlinear parabolic equations with nonlocal terms, Asymptot. Anal., 45 (2005), 301312.

[15] H. Crauel, A. Debussche, F. Flandoli, Random attractors, J. Dynam. Differential Equations, 9 (1997), 307-341.

[16] P. Freitas, Nonlocal reaction-diffusion equations, Differential equations with applications to biology (Halifax, NS, 1997), Fields Inst. Commun., 21, AMS, Providence, RL, 1999.

[17] J. Furter, M. Grinfeld, Local vs. nonlocal interactions in population dynamics, J. Math. Biol., 27 (1989), 65-80.

[18] J. Hale, Theory of Functional Differential Equations, Spring-Verlag, New York, 1977. 
[19] N. N. Krasovskiǐ, Stability of Motion, Applications of Lyapunov's Second Method of Differential Systems and Equations with Delay, Stanford University, 1963.

[20] N. I. Kavallaris, Explosive solutions of a stochastic non-local reaction-diffusion equation arising in shear band formation, Math. Meth. Appl. Sci., 38 (2015), 3564-3574.

[21] Y. J. Li, Y. J. Wang, The existence and asymptotic behavior of solutions to fractional stochastic evolution equations with infinite delay, J. Differential Equations, 266 (2019), 3514-3558.

[22] J. L. Lions, Quelques Méthodes de Résolution des Problèmes aux Limites Non Lineaires, Dunod, Paris, 1969.

[23] L. F. Liu, T. Caraballo, P. Marín-Rubio, Stability results for 2D Navier-Stokes equations with unbounded delay, J. Differential Equations, 265 (2018), 5685-5708.

[24] P. Marín-Rubio, J. Real, On the relation between two different concepts of pullback attractors for non-autonomous dynamical systems, Nonlinear Anal., 71 (2009), 3956-3963.

[25] P. Marín-Rubio, J. Real, J. Valero, Pullback attractos for a two-dimensional Navier-Stokes model in an infinite delay case, Nonlinear Anal., 74 (2011), 2012-2030.

[26] Z. Szymańska, C. Morales-Rodrigo, M. Lachowicz, M. A. J. Chaplain, Mathematical modelling of cancer invasion of tissue: the role and effect of nonlocal interaction, Math. Models Methods Appl. Sci., 19 (2009), 257-281.

[27] D. E. Tzaentis, Blow-up of radially symmetric solutions of a nonlocal problem modelling ohmic hearing, Electron. J. Diff. Eqns., 11 (2002), 1-26.

[28] X. Y. Evelyn Zhao, B. Hu, The impact of time delay in a tumor model, Nonlinear Anal. RWA, 51 (2020), 103015. 